Portland State University

PDXScholar

6-6-1975

\title{
The Dynamics of an HCP Crystal with a Substitutional Defect
}

Pramod C. Karulkar

Portland State University

Follow this and additional works at: https://pdxscholar.library.pdx.edu/open_access_etds

Part of the Atomic, Molecular and Optical Physics Commons Let us know how access to this document benefits you.

Recommended Citation

Karulkar, Pramod C., "The Dynamics of an HCP Crystal with a Substitutional Defect" (1975). Dissertations and Theses. Paper 2190.

https://doi.org/10.15760/etd.2187

This Thesis is brought to you for free and open access. It has been accepted for inclusion in Dissertations and Theses by an authorized administrator of PDXScholar. Please contact us if we can make this document more accessible: pdxscholar@pdx.edu. 
AN ABSTRACT OF THE THESIS OF Pramod C. Karulkar for the Master of Science in Physics presented June 6, 1975.

Title: The Dynamics of an HCP Crystal with a Substitutional Defect. APPROVED BY MEMBERS OF THE THESIS COMMITTEE:

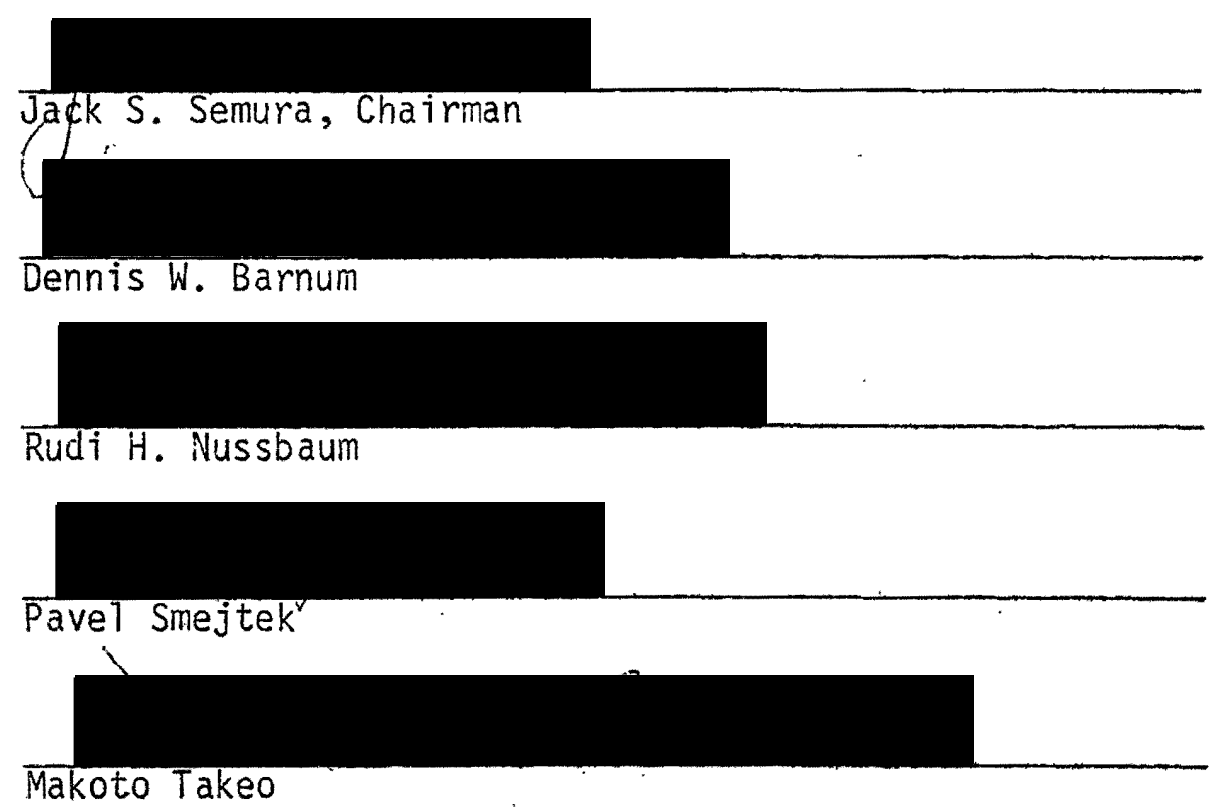

We examine the problem of the dynamics of a hexagonal close packed crystal with a single substitutional impurity. The effects of the mass and the force constant changes due to the introduction of the impurity atom are taken into account assuming nearest neighbor interactions under the harmonic approximation. Using Green's function and group theoretical methods, the equations of motion for the perturbed normal modes are obtained in an exact form. The calculations are performed by allowing for very general force constant changes which can have noncentral as well as central contributions. Further, by assuming central forces, we simplify the equations of motion for the 
nondegenerate perturbed normal modes in which the impurity atom participates and obtain the condition for the occurrence of a resonance or a localized mode, the defect amplitude of vibration $x_{\alpha}^{2}\left(0 ; \omega^{2}\right)$, and the defect mean square displacement $\left\langle u^{2}(0)>\right.$. The analytical expressions obtained in the central force approximation are of the same forms as those obtained by Mannheim and Cohen for cubic systems. 
DYNAMICS OF AN HCP CRYSTAL WITH

A SUBSTITUTIONAI DEFECT

\author{
by \\ PRAMOD C. KARULKAR
}

A thesis submitted in partial fulfillment

of the requirements for the degree of

\author{
MASTER OF SCIENCE \\ in \\ PHYSICS
}

\author{
Portland State University \\ 1975
}


TO THE OFFICE OF GRADUATE STUDIES AND RESEARCH:

The members of the Committee approve the thesis of Pramod C. Karulkar presented June 6, 1975.
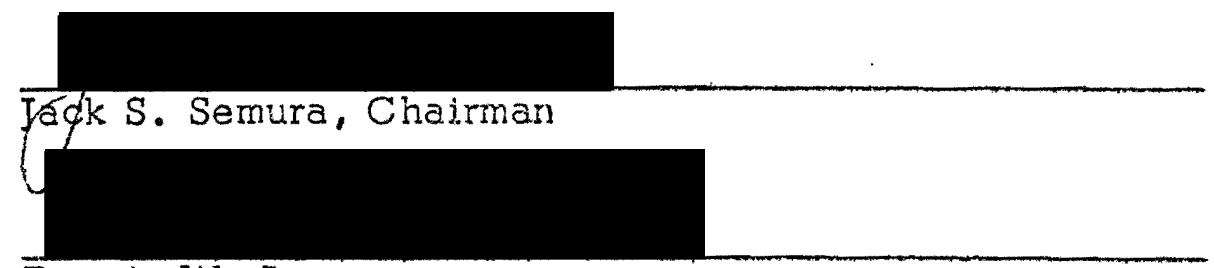

Dennis W. Barnum

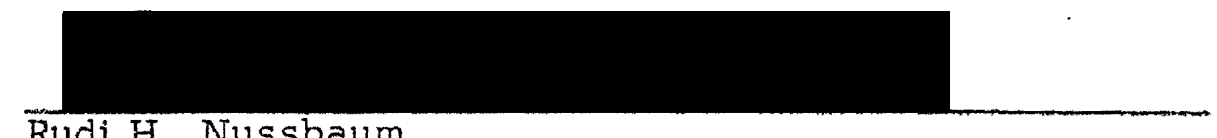

Rudi H. Nussbaum
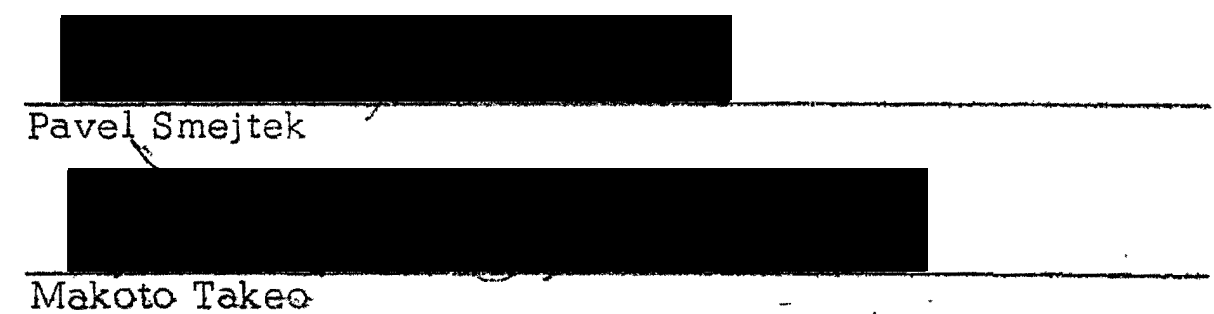

APPROVED:

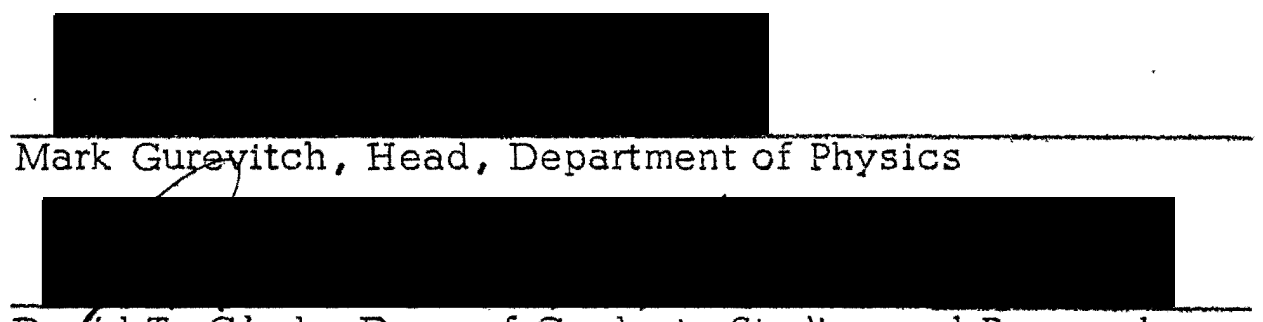

David T. Clark, Dean of Graduate Studies and Research

June 6,1975 


\section{ACKNOWLEDGEMENTS}

I am grateful to Dr. Jack $\mathrm{S}$. Semura for guidance and encouragement during the work on this problem. It is a pleasure to thank Professor R. H. Nussbaum for suggesting the problem. I wish to acknowledge very illuminating discussions with Professor Makoto Takeo, Dr. Simon S. Cohen, and Dr. Philip D. Mannheim. Further I wish to express appreciation to Professor R. P. Singh of the Indian Institute of Technology, Bombay, who introduced me to the elegance and usefulness of group-theoretical techniques. Thanks are also due to many persons, particularly to Mr. William J. Spurling of the Portland State University Computer Center, Mr. Shripad D. Tuljapurkar for help and encouragement, and Mrs. Frances Lancefield for the excellent typing.

Finally, I am indebted to many friends who made me feel at home in this place far from home. 
ACKNOWLEDGEMENTS ...................... i ii

IIST OF TABLES. . . . . . . . . . . . . . . v v

LIST OF FIGURES. . . . . . . . . . . . . . . . . . vi

LIST OF SYMBOLS . . . . . . . . . . . . . . . . vii

CHAPTER

I INTRODUCTION ................... 1

II MATHEMATICAL FORMULATION .......... 4

The Dynamics of a Perfect Crystal. . . . . . 4

The Dynamics of a Crystal with a

Substitutional Defect.......... 9

Transformation Properties of $\Phi, \delta L$, and $G$. $\quad 15$

Nature of Vibrations of the Perturbed Crystal . . 19

III AN HCP CRYSTAL WITH A DEFECT. . . . . . 21

Determination of Normal Modes ........ 21

The Defect Motion............. 29

IV DIAGONALIZATION ................ 34

$\mathrm{V}$ THE CASE OF CENTRAL FORCE INTERACTIONS . . . 48

Atomic Force Constants .......... 48

Nondegenerate Modes ........... 53

VI CONCLUSION AND RECOMMENDATIONS ...... 58

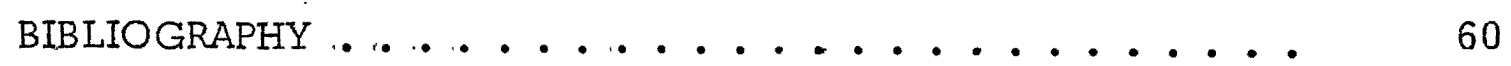

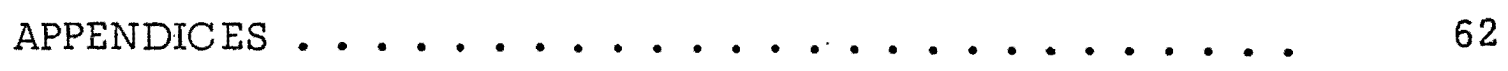




\section{LIST OF TABLES}

TABLE

PAGE

I Symmetry Operations of the Point Group $D_{3 h} \ldots \ldots 25$

II Determination of $X_{\mathrm{HCP}}(\mathrm{R}) \ldots \ldots \ldots 27$

III Character System for the Point Group $D_{3 h} \ldots . . . \quad 30$

IV Elements of the Matrix $g_{E_{22}^{\prime}} \ldots \ldots . \ldots . . . . . .41$

$\mathrm{V} \quad$ Elements of the Matrix $\delta I_{E_{22}^{\prime}} \ldots \ldots 44$

VI Displacements Belonging to the Various Normal

Modes of the Defect Cluster ........ 70

VII Bases for the Irreducible Representations of the

Point Group $D_{3 h^{*}} \ldots \ldots+\ldots$

VIII HCP Structures . . . . . . . . . 79 
FIGURE

PAGE

1. Impurity Cluster in an HCP Crystal. .........

23

2. Impurity Cluster Viewed Along the Main

Symmetry Axis ................. 24

3. Symmetry Operations of the Point Group $D_{3 h} \ldots \ldots 26$

4. Interatomic Distances in an HCP Impurity Cluster . . 52

5. Positions of hcp structures in the periodic table

of elements (indicated by circles) ........ 80 


\section{LIST OF SYMBOLS}

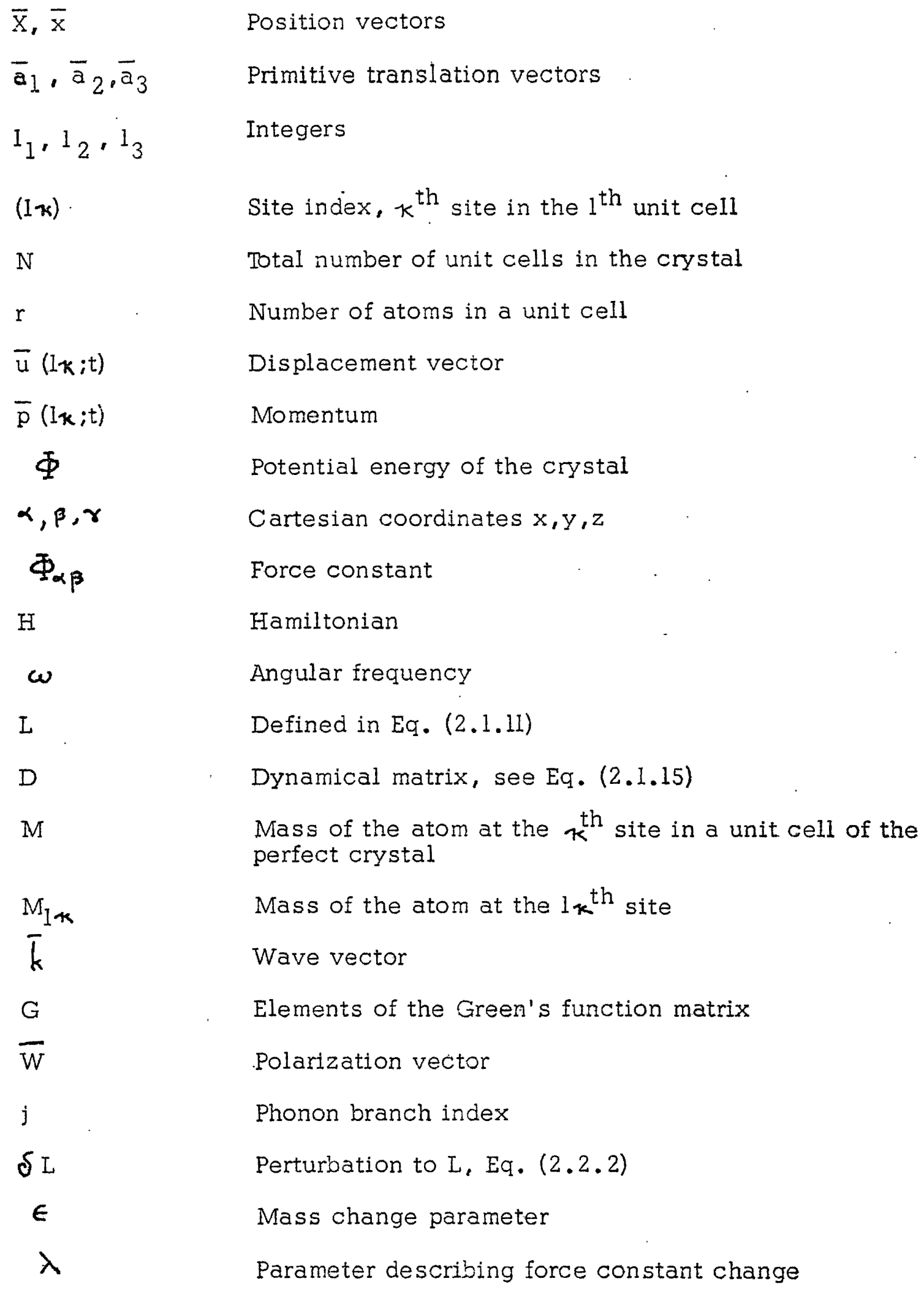

Dynamical matrix, see Eq. (2.1.15)

Mass of the atom at the $k^{\text {th }}$ site in a unit cell of the perfect crystal

Mass of the atom at the $1 x^{\text {th }}$ site

Wave vector

Elements of the Green's function matrix

Polarization vector

Phonon branch index

Perturbation to I, Eq. (2.2.2)

Mass change parameter

Parameter describing force constant change 
$z$

$n$

$\delta 1$

$g, G_{12}^{0}, G_{21}^{0}, G_{22}^{0}$

$u_{1}, u_{2}$

$\Delta\left(\omega^{2}\right)$

$\nu(\omega)$

$N\left(\omega^{2}\right)$

$\Delta N\left(\omega^{2}\right)$

\section{$\omega_{\text {LS }}$}

$\left|x_{\alpha}^{2}\left(0, \omega^{2}\right)\right|$

$\left\langle u_{\alpha}^{2}(0)\right\rangle$

$S$

$\bar{t}$

(Slt)

(S10)

$\mathrm{s}^{\mathrm{T}}$

THCP

$\chi_{\text {HCP }}$

$\mathrm{h}$

$1_{j}$

$a_{j}$

$\rho^{(j)}$
Number of nearest neighbours

$(z+1)$

Submatrix of $\delta I$

Submatrices of $\mathrm{G}^{0}$

Submatrices of $U$

Secular determinant

Frequency distribution function

Frequency spectrum

Change in the frequency spectrum

Frequency at a band edge

Amplitude of vibration of the defect atom

Niean square displacement of the defect

Matrix representation of a real orthogonal transformation

Translation vector

A space group operation

A point group operation

Transpose of $\mathrm{S}$

Total representation

Character of total representation

Number of elements in the point group, or Planck's constant

Dimensionality of the $j^{\text {th }}$ representation

Number of times $j^{\text {th }}$ irreducible representation occurs in $\Gamma_{\text {HCP }}$.

Projection operator for the $j^{\text {th }}$ irreducible representation. 
ix

$$
\begin{aligned}
& \mathrm{P}_{\mathrm{R}} \quad \text {. Symmetry operator } \\
& A_{n}^{\prime} \cdot A_{n}^{\prime r} \quad \text { One dimensional irreducible representations } \\
& \begin{array}{cl}
E^{\prime}, E^{n} & \text { Two dimension } \\
\psi & \text { Arbitrary fund } \\
\left\{\alpha_{n}\right\},\left\{\beta_{n}\right\} & \text { Basis vectors }
\end{array} \\
& \phi_{k x^{\prime}}(r) \quad \text { Pair potential energy in central force approximation } \\
& a, b, c, \eta_{i} \eta_{2} \text { see Figure } 4 \\
& \left.\begin{array}{l}
A, B, C, D ; F \\
g_{0}, g_{1}, g_{2}, g_{3}
\end{array}\right\} \text { See Es. (4.12) and (4.13) } \\
& \oint_{\mathrm{xx}}\left(\omega^{2}\right) \quad \text { See Eq. }(5.2 .13) \\
& S_{\mathbf{x x}}\left(\omega^{2}\right) . \quad \text { See Eq. } \cdot(5.2 .14)
\end{aligned}
$$




\section{CHAPTER I}

\section{INTRODUCTION}

Until recently the study of solids was limited to perfect solids which were assumed to have pure chemical composition and ideal periodic structure with certain symmetry properties. More realistic models are now receiving increased attention. One such problem which is being examined theoretically and experimentally is that of the dynamics of a crystal with defects. In particular, because of the discovery of the Mössbauer effect, attention has been focused on the dynamics of crystals with substitutional impurities. Although the investigations in this field were initiated by Lifshitz (1) in the years $1942-43$, they went unnoticed by the subsequent workers in the field for many years. Earlier calculations which took into account only isotopic impurities (8) are now being refined to include the changes in the force constants associated with the impurity atom. New mathematical techniques are available and more results on the lattice dynamical defect problem are being obtained. Various review articles published on the subject have considered the historical background and discussed different techniques in great depth (2-7).

The theoretical and experimental study of the dynamics of a crystal with impurities has two primary goals. One is the study of new effects, e.g. localized modes and resonance modes. The second is the use of impurity atoms as probes for studying the dynamical properties of the essentially perfect host crystal. The latter work includes Mọssbauer studies of samples with impurities. The mathematical 
difficulties involved in the analysis beyond a certain stage of simplification require introduction of more assumptions or the use of numerical methods.

Most of the work in this field has been limited to the systems of cubic symmetry, which is relatively easy to deal with $(8-14)$. The associated group theoretical analysis. is available in the literature (15). The present work examines the problem of the dynamics of a hexagonal close packed (HCP) crystal with a single substitutional defect. It is assumed that the introduction of the impurity does not distort the crystal structure. The calculation which is performed in the classical case assumes nearest neighbour interactions under the harmonic approximation: The effects of the mass change and the force constant change due to the introduction of the impurity are taken into account.

Chapter II deals with the general theory of the problem and discusses the use of the lattice Green's functions. The assumption of nearest neighbour interactions allows the reduction of the problem to a $39 \times 39$ matrix equation by using the matrix partitioning method (9). Dynamical quantities such as the density of states $\mathcal{\nu}(\omega)$ at the frequency $\omega$ and the defect' amplitude of vibration $\left|X_{\alpha}^{2}\left(0, \omega^{2}\right)\right|$ as a function of normal mode frequencies are introduced in this chapter.

Chapter III discusses the application of group theoretical techniques to the problem and the determination of the normal modes of the impurity cluster. Since the decomposition series for the total representation of an HCP impurity cluster and the basis vectors for the various irreducible representations are not available in the literature, a separate calculation was performed. The results appear in Chapter III and the 
detalls are in Appendix IV.

The equations of motion of the imperfect crystal in the matrix form have been block diagonalized in Chapter IV. It is important to note that the calculations through Chapter IV are performed by assuming very generalized force constant changes which can have noncentral as well as central contributions. A numerical calculation by the substitution of a large number of values for the Green's functions seems possible.

In order to obtain the expressions for the dynamical quantities in a closed analytical form, additional simplifications are necessary. To illustrate this, in Chapter $\mathrm{V}$ we make the central force approximation. Due to the lack of equivalence of the $x, y$, and $z$ axes in an HCP structure, the problem of the defect motion splits into two parts. One part expresses the solutions along the main symmetry axis and the other part corresponds to the axes perpendicular to it.

In order to maintain the continuity of the main text, much of the mathematical details are relegated to appendices.

The last appendix includes the periodic table of elements which indicates elemental crystals with the hexagonal structure. 


\section{CHAPTER II}

\section{MATHEMATICAI FORMULATION}

In this chapter we discuss the general method used for examining the problem of the dynamics of a crystal with a substitutional defect. After considering the equations of motion for a perfect crystal and for the same crystal with a substitutional impurity, we discuss the use of the Green's function techniques and the matrix partitioning method. A brief discussion on the transformation properties of the Green's function and the force constant is included. The nature of the modes of vibration of the imperfect crystal is considered qualitatively in the last section.

\section{THE DYNAMICS OF A PERFECT CRYSTAL}

Consider an arbitrary host crystal with $\mathrm{N}$ unit cells having $\mathrm{r}$ atoms per unit cell. The position of the $1^{\text {th }}$ unit cell relative to an origin located at some atom is given by:

$$
\overline{\mathrm{x}}(1)=1_{1} \overline{\mathrm{a}}_{1}+1_{2} \overline{\mathrm{a}}_{2}+1_{3} \overline{\mathrm{a}}_{3} \text {, }
$$

where $\bar{a}_{1}, \bar{a}_{2}$, and $\bar{a}_{3}$ are the three primitive translation vectors of the crystal; and $1_{1}, l_{2}$ and $1_{3}$ are integers which will be collectively represented by 1 . The position of the $k^{\text {th }}$ atom in the $1^{\text {th }}$. unit cell is given by

$$
\overline{\mathrm{X}}(1 k)=\overline{\mathrm{X}}(1)+\overline{\mathrm{X}}(x), \quad k=0,1, \ldots, r-1,
$$

where $\bar{X}(k)$ is the position of the $k^{\text {th }}$ atom with respect to the zeroth atom in a unit cell. We shall denote the displacement of the $1 x^{\text {th }}$ atom at time $t$ from its equilibrium position by $\bar{u}\left(l_{k} ; t\right)$ and the conjugate 
momentum by $\bar{p}\left(l_{k} ; t\right)$. The displacements are assumed to be periodic with the periodicity of the crystal:

$$
\bar{u}\left(l_{k} ; t\right)=\bar{u}((1+1) k ; t) \text {. }
$$

The potential energy of the host crystal can be expanded in Taylor's series in terms of the displacements of the atoms from their equilibrium positions:

$$
\begin{aligned}
\Phi^{*}= & \Phi_{0}^{0}+\sum_{l \kappa \alpha} \Phi_{\alpha}^{0}(1 k) u_{\alpha}(1 \kappa)+\frac{1}{2} \sum_{l k \alpha} \Phi_{\alpha \beta}^{0}\left(1 k ; l^{\prime} k^{\prime}\right) u_{\alpha}(1 k) u_{\beta}\left(l^{\prime} \kappa^{\prime}\right) \\
& + \text { Higher order terms, }
\end{aligned}
$$

Where $\alpha, \beta, \gamma$ represent the Cartesian axes $x, y$, or $z$; the superscript zero indicates that the quantity refers to a perfect crystal and the subscript zero indicates that the quantity is evaluated with all the atoms at their equilibrium positions. The coefficients $\Phi_{\alpha}^{0}\left(l_{k}\right)$ and $\Phi_{\alpha \beta}^{0}\left(l_{k} ; l^{\prime} k^{\prime}\right)$ are defined as:

$$
\begin{aligned}
& \Phi_{\alpha}^{0}(1 k)=\left[\partial \Phi^{0} / \partial u_{\alpha}(l k)\right]_{0}, \\
& \Phi_{\alpha \beta}^{0}\left(l k_{j} l^{\prime} k^{\prime}\right)=\left[\partial \Phi^{0} / \partial u_{\alpha}(l k) \partial u_{\beta}\left(l^{\prime} k^{\prime}\right)\right]_{0} .
\end{aligned}
$$

Since the choice of $\Phi^{\circ}$ in Eq. (2.1.4) is arbitrary, it can be taken to be zero; $\Phi_{\alpha}^{0}$. vanishes for all $l_{\kappa}$ because the potential energy $\Phi^{*}$ is minimum when all the atoms are at their respective equilibrium positions. By retaining only $\Phi_{\alpha \beta}^{0}$ and neglecting all the higher order terms, we write the hamiltonian of the host crystal in the harmonic approximation as

$$
H=\frac{1}{2} \sum_{l k \alpha} M_{k} \dot{u}_{\alpha}^{2}\left(l_{k} ; t\right)+\frac{1}{2} \sum_{\substack{l k \alpha \\ l^{\prime} k^{\prime} \beta}} \Phi_{\alpha \beta}^{0}\left(1 k ; l^{\prime} k^{\prime}\right) u_{\alpha}(1 k) u_{\beta}\left(l^{\prime} k^{\prime}\right)
$$

The coefficients $\Phi_{\alpha \beta}^{0}$ are called the second order force constants. It is well known that neglecting the higher order terms in Eq.(2.1.4) is equivalent to neglecting the phonon-phonon interactions which lead to 
a renormalization and damping of the phonon frequency spectrum obtained th in the harmonic approximation. The equation of motion of the $1 k$ atom of the perfect crystal follows immediately:

$$
\operatorname{Mu}_{k^{\prime}}(I k ; t)=-\sum_{i^{\prime} x^{\prime} \beta} \Phi_{\alpha \beta}^{0}\left(1 k ; I^{\prime \prime} k^{\prime}\right) u_{\beta}\left(I^{\prime} k^{\prime}\right)
$$

We assume a harmonic time dependence for the displacement co-ordinates:

$$
u_{\alpha}(l k ; t)=u_{\alpha}(1 k) \exp (1 \omega t)
$$

This solution implies that all the atoms are vibrating with the same frequency $\boldsymbol{\omega}$ which is called a normal mode frequency. Such solutions are called normal mode solutions. Any arbitrary vibration in the crystal is a superposition of the normal modes. Now Eq. (2.1.8) reduces to the equation of motion for the time independent amplitude $u_{\alpha}(1 \star)$ :

$$
\sum_{\ell^{\prime} k^{\prime} \beta}\left[M_{k} \omega^{2} \delta_{11^{\prime}} \delta_{k k^{\prime}} \delta_{\alpha \beta}-\Phi_{\alpha \beta}^{0}\left(1 k i 1^{\prime} k^{\prime}\right)\right] u_{\beta}\left(1^{\prime} k^{\prime}\right)=0 .
$$

We can write this equation more compactly in a matrix form:

$$
\mathrm{Lu}=0,
$$

where $\mathrm{L}$ is a $3 \mathrm{Nr} \times 3 \mathrm{Nr}$ matrix with the rows and the columns labelled by the triple index $\{1 k \alpha\}$. The expression in the braces in Eq. (2.1.10) is the $\left(1 \kappa \alpha ; l^{\prime} k^{\prime} \beta\right.$ ) element of the matrix L. The matrix $u$ is a column vector whose elements are the displacement amplitudes $\left\{u_{\alpha}(1 k)\right\}$. Eq. (2.1.11) has $3 \mathrm{Nr}$ solutions which correspond to $3 \mathrm{Nr}$ normal mode frequencies. All the normal mode frequencies need not be distinct. The degeneracies of the normal mode frequencies need a separate consideration.

Equation (2.1.8) represents a set of $3 \mathrm{Nr}$ equations and for a crystal, $\mathrm{N}$ is infinitely large. This problem of solving infinite numbers of 
simultaneous equations can be simplified by using the periodicity of the crystal. The invariance of the potential energy of a crystal under a crystal translation operation lends to the result:*

$$
\Phi_{\alpha \beta}^{0}\left(l k_{j} l^{\prime} k^{\prime}\right)=\Phi_{\alpha \beta}^{0}\left(\left(l-l^{\prime}\right) k_{j} \circ k^{\prime}\right)=\Phi_{\alpha \beta}^{0}\left(0 k_{j}\left(l^{\prime}-l\right) k^{\prime}\right) .
$$

If we choose as a solution to Eq. (2.1.8) a function of the form

$$
u_{\alpha}\left(l_{k} ; t\right)=M_{k}^{1 / 2} u_{\alpha}(\dot{k}) \exp [-i \omega t+i \bar{k} \cdot \vec{x}(1)] \text {, }
$$

where $\bar{k}$ is the wave vector and $u_{\alpha}(k)$ is independent of 1 , and substitute this expression into Eq. $(2.1 .8)$ we find that:

$$
\omega^{2} u_{\alpha}(k)=\sum_{k \beta} D_{\alpha \beta}^{0}\left(k k^{\prime} \mid \bar{k}\right) u_{\beta}\left(x^{\prime}\right),
$$

where the elements of the matrix $D^{0}(\bar{k})$, called the Fourier transformed dynamical matrix are given by

$$
D_{\alpha \beta}^{0}\left(k k^{\prime} \mid \bar{k}\right)=\left(M_{k} M_{k^{\prime}}\right)^{1 / 2} \sum_{l^{\prime}} \Phi_{\alpha \beta}^{0}\left(l k_{j} l^{\prime} k^{\prime}\right) \exp \left[-i \bar{k} \cdot\left(\bar{x}(l)-\bar{x}\left(l^{\prime}\right)\right)\right] \text {. }
$$

We have written the left hand side of Eq. (2.1.15) independent of 1 and $l^{\prime}$ because as shown in Eq. (2.1.12), $\Phi_{\alpha \beta}\left(\lambda_{k_{i}}\left(k^{\prime}\right)\right.$ does not depend on 1 and $1^{\prime}$ separately.

Thus the problem of the perfect crystal is reduced to a set of $3 \mathrm{r}$ linear homogeneous equations in $3 r$ unknowns, $\left\{u_{\alpha}\left(l_{k}\right)\right\}$. The condition that equations (2.1.14) have a nontrivial solution is that the determinant of the coefficients vanish:

$$
\left|D_{\alpha \beta}\left(k k^{\prime} \mid \bar{k}\right)-\omega^{2} \delta_{\alpha \beta} \delta_{k k^{\prime}}\right|=0 \text {. }
$$

For each $\vec{k}$ there are $3 r$ solutions $\omega_{j}^{2}(\bar{k})$ where $j=1,2,-\cdots 3 r$.

* Some important properties of the force constants are discussed in the third section of this chapter. 
This shows that the relation

$$
\omega=\omega_{j}(\bar{k})
$$

which is known as the dispersion relation has 3r.branches labelled by $3 r$ values of $j$.

Before concluding this section we introduce the Green's function matrix for a perfect crystal:

$$
\begin{aligned}
G_{\alpha \beta}^{0}\left(1 k ; l^{\prime} k^{\prime},\right. & \left.\omega^{2}\right)=\left[L_{\alpha \beta}^{-1}\left(1 k i l^{\prime} k^{\prime} ; \omega^{2}\right)\right] \\
& =\frac{1}{N\left(M_{k} M_{k^{\prime}}\right)^{1 / 2}} \sum_{\bar{k} j} \frac{\bar{W}_{\alpha}\left(k \mid k_{j}\right) \bar{W}_{\beta}^{*}\left(k^{\prime} \mid k_{j}\right)}{\omega^{2}-\omega_{j}^{2}(\bar{k})} \\
& x \exp \left\{i k \cdot\left[\bar{X}\left(l_{k}\right)-\bar{X}\left(l^{\prime} k^{\prime}\right)\right]\right\},
\end{aligned}
$$

where $\bar{W}\left(k \mid k_{j}\right)$ is the polarization vector of the $k^{\text {th }}$ atom participating in the normal mode characterized by the wave vector $\bar{k}$ and the phonon branch index $j$. The allowed $\vec{k}$ values are determined by the boundary conditions and the sum is taken over the first Brillouin zone. The Green's function satisfies the same boundary conditions as $\left\{u_{\alpha}(1 k)\right\}$

The definition of $\mathrm{G}_{\alpha \beta}^{0}\left(1 k, l^{\prime} k^{\prime}, \omega^{2}\right)$ can be written as

$$
\begin{aligned}
& \sum_{l_{2} k_{2} \beta} L_{\alpha \beta}\left(I_{1} k_{1} ; I_{2} k_{2} ; \omega^{2}\right) G \quad\left(l_{2} k_{2} ; I_{3} \quad k_{3} ; \omega^{2}\right) \\
& =\delta_{\alpha \gamma} \delta_{1_{1} 1_{3}} \delta_{k_{1} k_{3}} .
\end{aligned}
$$

Substituting the expression for $L_{\alpha \beta}$ from $\mathrm{Eq} .(2.1 .10)$ and rearranging, we obtain a summation relation for the elements of the Green's function matrix: 
9

$$
\begin{aligned}
& \sum_{1_{2} k_{2} \beta} \Psi_{\alpha \beta}^{0}\left(1_{1} k_{1} ; 1_{2} k_{2}\right) G_{\beta \gamma}^{0}\left(1_{2} k_{2} ; 1_{3} k_{3} ; \omega^{2}\right) \\
& =-\delta_{\alpha \gamma} \frac{1}{N} \sum_{\bar{k}} \exp \left\{i \bar{k} \cdot\left[\bar{x}\left(1, k_{1}\right)-\bar{X}\left(1_{3} k_{3}\right)\right]\right\} \\
& +M \omega^{2} G_{\alpha \beta}^{\circ}\left(l_{1} k_{1} ; 1_{3} k_{3} ; \omega^{2}\right) .
\end{aligned}
$$

II. THE DYNAMIC S OF A CRYSTAL WITH A SUBSTITUTIONAL DEFECT

If one of the atoms in the host crystal considered in the first section is replaced by an impurity atom, we can rewrite the Ens. (2.1.4) to (2.1.11) for this perturbed crystal by dropping out the superscript zero. The equations of motion of the time independent amplitudes of the atoms of the perturbed crystal can be written in the form:

$$
\sum_{\substack{l^{\prime} k^{\prime} \beta \\=0}}\left[M_{1 k} \omega^{2} \delta_{11^{\prime}} \delta_{k k^{\prime}} \delta_{\alpha \beta}-\Phi_{\alpha \beta}\left(1 k_{;} l^{\prime} k^{\prime}\right)\right] u_{\beta}\left(1^{\prime} k^{\prime}\right)
$$

where $M_{1 k}$ is the mass of the atom at the site $(1 k)$ and $\Phi_{\alpha \beta}$ are the atomic force constants for the perturbed crystal. We can write Eq. (2.2.1) in a more compact form:

$$
(I-\delta I) U=0 \text {, }
$$

where $\delta I$ describes the effects of the presence of the impurity on the crystal. The elements of the matrix $\delta$ I are:

$$
\begin{aligned}
& \delta_{L_{\alpha \beta}}\left(I_{k i 1^{\prime} k^{\prime}} ; \omega^{2}\right)=\left[\omega^{2}\left(M_{k}-M_{1 k}\right) \delta_{11^{\prime}} \delta_{k k^{\prime}} \delta_{\alpha \beta}\right] \\
& +\left[\Phi_{\alpha \beta}\left(1 k ; l^{\prime} k^{\prime}\right)-\Phi_{\alpha \beta}^{0}\left(1 k ; l^{\prime} k^{\prime}\right)\right] \\
& =\left[\in M_{k} \omega^{2} \delta_{11^{\prime}} \delta_{k k^{\prime}} \delta_{\alpha \beta}\right]-\left[\lambda_{\alpha \beta}\left(1 k i 1^{\prime} k^{\prime}\right) \Phi_{\alpha \beta}^{0}\left(1 k i l^{\prime} k^{\prime}\right)\right] .
\end{aligned}
$$


We have introduced the mass change parameter $\epsilon$ and the force constant change parameter $\lambda_{\alpha \beta}$ which are defined as:

$$
\begin{aligned}
& \epsilon=\left[M_{k}-M_{1^{k}}\right] / M_{k}, \\
& \lambda_{\alpha \beta}\left(l_{k} ; l^{\prime} k^{\prime} \cdot\right)=1-\left[\Phi_{\alpha \beta}\left(1 k ; l^{\prime} k^{\prime}\right) / \Phi_{\alpha \beta}^{0}\left(1 k_{i} 1^{\prime} k^{\prime}\right)\right] .
\end{aligned}
$$

The condition for solvability for the system of equations given by Eq. $(2.2 .2)$ is

$$
|L-\delta L|=0 \text {, }
$$

the solutions of which are the normal mode frequencies $\left\{\omega_{s}\right\}$ of the perturbed crystal. The determinant in Eq. $(2.2 .6)$ has infinite dimensionality for very large N. For reducing the problem to a reasonable size one assumes nearest neighbour interactions. According to this assumption, the presence of an impurity is felt only by its nearest neighbours. In this approximation

$$
\begin{aligned}
& \delta L_{\alpha \beta}\left(l_{k} ; 1 \kappa ; \omega^{2}\right) \neq 0 \text { if } I \kappa \text { refers to the impurity and its } \\
& \delta L_{\alpha \beta} \quad\left(1 k ; l^{\prime} k^{\prime} ; \omega^{2}\right) \neq 0 \text { when one of the sites }(1 k) \text { and }\left(l^{\prime} k^{\prime}\right) \\
& \text { refers to the defect and the other } \\
& \text { refers to a nearest neighbour, } \\
& \delta L_{\alpha \beta} \cdot\left(l_{k} ; l^{\prime} k^{\prime} ; \omega^{2}\right)=0 \text { otherwise. }
\end{aligned}
$$

For a crystal with coordination number $z$, the number of nonzero elements will be $(3 z+1)$.

By using the definition of the pure crystal Green's function given in Eq. (2.1.18), we rewrite Eq. (2.2.2) as

$$
\left(I-G^{0} \delta L\right) U=0 \text {. }
$$

This is a $3 \mathrm{Nr}$ dimensional equation. We use the matrix partitioning 
method introduced by Lehmann and De Wames (9). Under the assumption of nearest neighbour interactions, by suitable labelling of the rows and the columns, $\delta \mathrm{L}$ can be written in the partitioned form:

$$
\delta L=\left[\begin{array}{ccc}
\delta l & 1 & 0 \\
- & -1 & -- \\
0 & 1 & 0
\end{array}\right],
$$

where $\delta 1$ is a $3(z+1) \times 3(z+1)$ submatrix whose rows and columns are labelled by the indices $(1 k \alpha)$ and $\left(l^{\prime} k^{\prime} \beta\right)$ of the impurity site and its nearest neighbours. The subspace spanned by the nonzero part of $\delta L$ will be called "the subspace of $\delta 1$ " in the discussion which follows: Similarly we can partition the matrices $G^{0}$ and $U$ as:

$$
G^{0}=\left[\begin{array}{ccc}
g & G_{12}^{0} \\
\hdashline G_{21}^{0} & G_{22}^{0}
\end{array}\right], \quad U=\left[\begin{array}{c}
u_{1} \\
-u_{2}
\end{array}\right]
$$

where $g$ and $u_{1}$ are in the subspace of $\delta 1$. The column vector $u_{2}$ includes the displacements of the atoms in the cluster formed by the impurity atom and its nearest neighbours. This will be referred to as the impurity cluster. The substitution of Eqs. (2.2.8) and (2.2.9) in Eq. (2.2.7) gives:

$$
\begin{aligned}
& u_{1}=g \delta_{1} u_{1}, \\
& u_{2}=G_{21}^{0} \delta_{1} u_{1} .
\end{aligned}
$$

Eq. (2.2.10) describes the motion of the impurity cluster and Eq. (2.2.11) describes the motion of the rest of the crystal. The condition for Eq. $(2.2,10)$ to have nontrivial solutions is:

$$
\Delta\left(\omega^{2}\right)=|I-g \delta 1|=0 \text {. }
$$


The solutions of this equation give the normal mode frequencies of the impurity cluster. It can be shown that these are the only normal modes which are perturbed by the introduction of the impurity (17). The study of the dynamics of the perturbed crystal matnly concerns the perturbed modes. Hence in the remaining part of this work we shall be interested in $\mathrm{Eq} .(2,2,12)$

Before concluding this section we introduce some dynamical quantities.

We define the frequency distribution function. $\nu(\omega)$ by the relation (18).

$$
\nu(\omega) d \omega=\lim _{N \rightarrow \infty} \frac{N(\omega) d \omega}{N},
$$

where $N(\omega)$ d $\omega$ is the number of modes in the interval $(\omega, \omega+d \omega)$. The frequency distribution function is sometimes called the density of states function and fulfils the normalization condition

$$
\int_{0}^{\infty} V(\omega) d \omega=3 r
$$

The concept of the frequency spectrum $N\left(\omega^{2}\right)$ is sometimes very useful. * It is defined in such a way that $N\left(\omega^{2}\right) \mathrm{d} \omega^{2}$ is the fraction of the normal modes whose squared frequencies lie in the interval $\left(\omega^{2}, \omega^{2}+d \omega^{2}\right)$ in the limit as $d \omega^{2}$ tends to zero (4). The spectrum $N\left(\omega^{2}\right)$ is related to the distribution function $V(\omega)$ by the relation

$$
\nu(\omega)=2 \omega N\left(\omega^{2}\right)
$$

*The standard notation for the density of states function and the frequency spectrum is $g(\omega)$ and $G\left(\omega^{2}\right)$ respectively. We have used the notation $\nu(\omega)$ and $N\left(\omega^{2}\right)$ to avoid confusion between these functions and the Green's functions. 
The frequency spectrum can be expressed as

$$
N\left(\omega^{2}\right)=(1 / 3 N r) \sum_{s} \delta\left(\omega^{2}-\omega_{s}^{2}\right),
$$

where the summation is taken over all the normal mode frequencies. It can be shown that the change in the frequency spectrum due to the introduction of an impurity atom is given by:

$$
\begin{aligned}
N\left(\omega^{2}\right) & =N\left(\omega^{2}\right)-N^{0}\left(\omega^{2}\right) \\
& =(1 / 3 N r \pi) \operatorname{Im} \frac{d}{d \omega^{2}}\left[\ln \Delta\left(\omega^{2}-i o\right),\right]
\end{aligned}
$$

where $\Delta\left(\omega^{2}\right)$ is defined in Eq. (2.2.12) and $\omega$ lies in the frequencies accessible to the normal modes of the perfect crystal. The frequency spectrum of the perturbed crystal has a $\delta$-function character when localized modes are present. The change in the frequency spectrum in this case is given by

$$
\Delta N\left(\omega^{2}\right)=(1 / 3 N r) \sum_{s}^{\prime}\left[\delta\left(\omega^{2}-\omega_{s}^{2}\right)-\delta\left(\omega^{2}-\omega_{L_{s}}^{2}\right)\right]
$$

where the prime on the sum means that the sum extends over the localized modes only, degenerate modes being counted as many times as they occur, and $\omega_{\text {Ls }}$ is the frequency of the band edge into which the localized modes return as the perturbation due to the impurity atom tends to zero.

From the definition of the Green's function for the perfect lattice given in Eq:(2.1.18)we obtain

$$
G_{\alpha \alpha}^{0}\left(0 ; 0 ; \omega^{2}\right)=\frac{1}{N M} \sum_{\bar{k} j} \frac{W_{\alpha}(0 \mid \bar{k} j) w_{\beta}^{*}(0 \mid \bar{k} j)}{\omega^{2}-\omega_{j}^{2}(\bar{k})} .
$$


For cubic systems for which the three co-ordinate axes are equivalent, after summation over $\alpha$, above equation reduces to

$$
\operatorname{Im} G_{x x}^{0}\left(0 ; 0 ; \omega^{2}\right)=\frac{\pi}{M} N^{0}\left(\omega^{2}\right)
$$

In case of an HCP crystal we can not write such a relation since the co-ordinate axes are not equivalent.

The Green's function matrix for the perturbed crystal can be written as

$$
\begin{aligned}
G & =(L-\delta L)^{-1} \\
& =\left(1-G^{0} \delta L\right)^{-1} G^{0} .
\end{aligned}
$$

The Green's function for the perturbed crystal is related to the amplitude of vibration $\left|\chi_{\alpha}^{2}\left(0 ; \omega^{2}\right)\right|$ of the defect atom vibrating in the perturbed mode $\omega$ in the direction $\alpha$ through the relation $(13,22)$ :

$$
\operatorname{Im} G_{\alpha \alpha}\left(0 ; 0 ; \omega^{2}\right)=3 \mathrm{MN}\left|\chi_{\alpha}^{2}\left(0 ; \omega^{2}\right)\right| \operatorname{Img} \alpha \alpha\left(0 ; 0 ; \omega^{2}\right)
$$

from which we obtain

$$
\left|\chi_{\alpha}^{2}\left(0 ; \omega^{2}\right)\right|=\frac{\operatorname{Im} G_{\alpha \alpha}\left(0 ; 0 ; \omega^{2}\right)}{3 M N \operatorname{Img} g_{\alpha \alpha}\left(0 ; 0 ; \omega^{2}\right)} .
$$

The mean square displacement $\left\langle u_{\alpha}^{2}(0)\right\rangle$ of the defect which is very important in Mös sbauer studies is related to $\left|\chi_{\alpha}^{2}\left(0 ; \omega^{2}\right)\right|$. It can be shown that (22):

$$
\left\langle u_{\alpha}^{2}(0)\right\rangle=\frac{3 \hbar M N}{\pi} \int_{0}^{\infty} d \omega \operatorname{coth}\left(\frac{1}{2} \hbar \beta \omega\right)\left|\chi_{\alpha}^{2}\left(0 ; \omega^{2}\right)\right| \operatorname{Im} g_{\alpha \alpha}\left(0 ; 0 ; \omega^{2}\right) .
$$

The matrices on the right hand side of Eq. (2.2.20) can be obtained in terms of the linearly independent elements of $G^{0}$ and $\delta L$. The inverse of the matrix $\left(1-G^{0} \delta L\right)$ can be obtained after making the 
nearest neighbour approximation and subsequent block diagonalization of the submatrix $(1-g \delta 1)$. The results of the calculations which we have presented in the following chapters can be used in Eqs. (2.2.22) and $(2,2,23)$.

\section{TRANSFORMATION PROPERTIES OF $\Phi, \delta L$, AND G}

The matrices $\Phi, \delta I$ and $G$ are not explicitly known. The properties of these matrices which are useful in reducing the number of independent matrix elements are summarized in this section (4).

The atomic force constants are symmetric in their arguments:

$$
\Phi_{\alpha \beta}^{0}\left(1 k i l^{\prime} k^{\prime}\right)=\Phi_{\beta \alpha}^{0}\left(1^{\prime} k^{\prime} ; 1 k\right) .
$$

The invariance of the force on an atom against a rigid body translation of the crystal leads to the condition:

$$
\sum_{l^{\prime} k^{\prime}} \Phi_{\alpha \beta}^{0}\left(l \cdot k_{j} l^{\prime} k\right)=0
$$

The invariance of potential energy against an infinitesimal rigid body rotation of the crystal results in the condition:

$$
\begin{aligned}
\sum_{l^{\prime} k^{\prime}}\left\{\Phi_{\alpha \beta}^{0}\left(1 k ; 1^{\prime} k^{\prime}\right)\right. & \left.X_{\gamma}\left(1^{\prime} k^{\prime}\right)\right\} \\
& =\sum_{l^{\prime} k^{\prime}}\left\{\Phi_{\alpha \gamma}^{0}\left(1 k_{i} 1^{\prime} k^{\prime}\right) x_{\beta}\left(1^{\prime} k^{\prime}\right)\right\} \quad(2.3 .3)
\end{aligned}
$$

The three conditions in Eqs. $(2.3 .1)$ to $(2.3 .3)$ do not depend on the periodicity of the lattice. They are the basic property of a system of particles with the potential energy expressible as a quadratic function of the displacements of the particles from the equilibrium positions. Hence they are also valid for a crystal with a point defect. 
For a perfect crystal in which the atomic displacements satisfy the periodic boundary conditions, the invariance of the crystal under a rigid body displacement through one of its translation vector requires that

$$
\begin{aligned}
& \Phi_{\alpha \beta}^{0}\left(1 * ; I^{\prime} k^{\prime}\right) \text { should depend on the difference between } 1 \text { and } 1^{\prime} \text { : } \\
& \Phi_{\alpha \beta}^{0}\left(l_{k_{j}} l^{\prime} x^{\prime}\right)=\Phi_{\alpha \beta}^{0}\left(\left(l-l^{\prime}\right) k_{i} 0 x^{\prime}\right)=\Phi_{\alpha \beta}^{0}\left(0 x_{j}\left(l^{\prime}-l\right) x^{\prime}\right) .
\end{aligned}
$$
is the way in which they transform under an operation of the space group of the crystal. The most general transformation is denoted by the symbol $(S \mid \bar{t})$, where $S$ is the $3 \times 3$ matrix representation of a real orthogonal transformation, a proper or improper rotation, while $\bar{t}$ is a vector through which the crystal is translated. When $\bar{t}$ is a crystal translation vector, the group of operations is called "symmorphic". When $\bar{t}$ is a fraction of a crystal translation vector, the group is called "nonsymmorphic". The fractional translations are associated with screw axes or glide planes. If a space group operation $(S \mid \bar{t})$ takes the atom at the site $\left(l_{k}\right)$ to an equivalent site (LK), we can express the operation as:

$$
\bar{X}(I K)=(S \mid \bar{t}) \bar{X}\left(I_{K}\right)=S \bar{X}\left(l_{k}\right)+\bar{t} .
$$

In the following discussion we shall adopt the convention that the small letter $(1 \times)$ will denote the initial position of the atom and the capital letters (LK) will represent the final position of the same atom after the symmetry operation under consideration. Equation (2.3.5) can be written in the component form:

$$
x_{\alpha}(L K)=\sum_{p} S_{\alpha p} X_{p}(I x)+\bar{t} \text {. }
$$

The Invartance of the potential energy of a perfect crystal under the 
space group operation $(S \mid \bar{t})$ yields the law of transformation for the atomic force constants:

$$
\Phi_{\alpha_{1} \alpha_{2}}^{0}\left(I_{1} K_{1} ; L_{2} K_{2}\right)=\sum_{\beta_{1} \beta_{2}} S_{\alpha_{1} \beta_{1}} S_{\alpha_{2} \beta_{2}} \Phi_{\beta_{1} \beta_{2}}^{0}\left(l_{1} k_{1} ; I_{2} k_{2}\right),
$$

which can be written in a matrix form

$$
\Phi^{0}\left(I_{1} K_{l} ; L_{2} K_{2}\right)=S \Phi^{\circ}\left(I_{1} k_{1} ; I_{2} k_{2}\right) S^{T},
$$

where all the matrices are $3 \times 3$ and $S^{T}$ is the transpose of $S$. We note that apart from a possible interchange of sublattices the force constants transform as the components of a second rank tensor.

For a crystal with a single substitutional impurity, the functional form of the atomic interactions remains the same as that for the atoms in the perfect crystal. Hence the functional forms of the total potential energy $\Phi$ and the atomic force constants $\Phi_{\alpha \beta}$ in the pert urbed case are the same as those for the perfect crystal. The only difference we have to note while dealing with the perturbed crystal is the loss of the translational symmetry. In this case we have to use the point group operations $(S \mid O)$ which keep the defect atom unshifted. Hence the Eqs. (2.3.1) to $(2.3 .7)$ can be rewritten for the perturbed crystal by removing the superscript zero and remembering that we are using the point group operations:

$$
\begin{gathered}
\Phi_{\alpha \beta}\left(l_{k}, l^{\prime} k^{\prime}\right)=\Phi_{\beta \alpha}\left(l^{\prime} k^{\prime}, l k\right), \\
\sum_{l^{\prime} k^{\prime}} \Phi_{\alpha \beta}\left(l_{k}, l^{\prime} k^{\prime}\right)=0, \\
\sum_{l^{\prime} k^{\prime}} \Phi_{\alpha \beta}\left(l k, l^{\prime} k_{j}^{\prime}\right) X_{\gamma}\left(l^{\prime} k^{\prime}\right)=\sum_{l^{\prime} k^{\prime}} \Phi_{\alpha \gamma}\left(l_{k} l^{\prime} l_{k}^{\prime}\right) X_{\beta}\left(l^{\prime} k^{\prime}\right),
\end{gathered}
$$




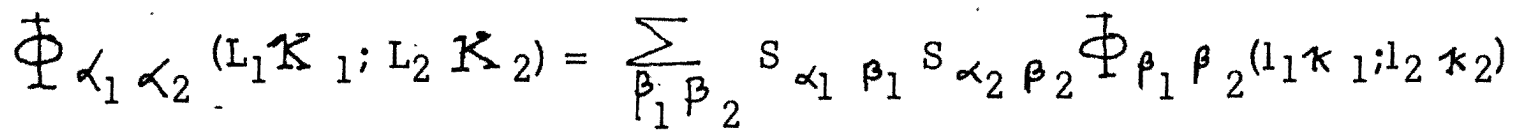

$$
\begin{aligned}
& \Phi\left(L_{1} K_{1} ; L_{2} K_{2}\right)=s \Phi\left(l_{1} k_{1} ; I_{2} k_{2}\right) s^{T}
\end{aligned}
$$

From the form of $\delta L$ in Eq. (2.2.3) it follows that $\delta L \alpha \beta$ satisfies the transformation law:

$$
L_{\alpha_{1} \alpha_{2}}\left(L_{1} K_{2} ; L_{2} K_{2}\right)=\sum_{\beta_{1} \beta_{2}} S \alpha_{1} \beta_{1} S \alpha_{2} \beta_{2} L_{\beta_{1} \beta_{2}}\left(1_{1} k_{1} ; l_{2} k_{2}\right),
$$

which can be written in a matrix form as:

$$
L\left(L_{1} K_{1} ; L_{2} K_{2}\right)=S \delta L\left(l_{1} K_{1} ; l_{2} K_{2}\right) s^{T} .
$$

It can be shown that the Green's functions for a perfect crystal transform according to the law (4)

$$
G_{\alpha_{1} \alpha_{2}}^{0}\left(L_{1} K_{1} ; L_{2} K_{2} ; \omega^{2}\right)=\sum_{\beta_{1} \beta_{2}} S_{\alpha_{1} \beta_{1}} S_{\alpha_{2} \beta_{2}} G_{\beta_{1} \beta_{2}}^{0}\left(1_{1} \kappa_{1} ; l_{2} \kappa_{2} ; \omega^{2}\right),
$$

which can be written in a compact form as:

$$
G^{0}\left(L_{1} K_{1} ; L_{2} K_{2} ; \omega^{2}\right)=S G^{0}\left(1_{1} \pi_{1} ; 1_{2} \pi_{2} ; \omega^{2}\right) s^{T} .
$$

For obtaining the solutions to Eq. $(2.2 .12)$ we must have the matrices $g$ and $\delta l$ which are submatrices of $G^{0}$ and $\delta L$. By using the transformation laws discussed above, we can determine the elements of $g$ and $\delta \mathrm{l}$ in terms of their respective linearly independent elements. Appendix III illustrates the use of the transformation laws. 
IV. THE NATURE OF VIBRATIONS OF THE PERTURBED CRYSTAL

In this section we shall briefly comment on the nature of the solutions to Eqs. $(2 \cdot 2 \cdot 10)$. The mathematical verification of the various statements is beyond the scope of this presentation because of the limitations of the theoretical background developed up to this point in this chapter.

The displacement amplitudes for atoms vibrating in a perturbed mode whose frequency lies above the maximum frequency of the unperturbed crystal or in a gap in its frequency spectrum decay faster than exponentially with increasing distance from the impurity atom. For this reason such modes are called localized modes. If the frequency of a localized mode lies in a gap in the frequency spectrum of the host crystal, the mode is referred to as a gap mode. The rate of decay of the displacement amplitude is larger when the frequency of the localized mode $\omega$ is at a greater distance from the band edge. In general, it is found that localized modes do not exist for arbitrary changes in the mass and the force constants associated with the impurity atom. Their occurrence depends on the detailed magnitudes of the changes of the mass and the force constants. In the case of an isotopic impurity where one can assume no changes of force constants, localized modes occur if the mass of the impurity atom is less than that of the host atom it substitutes by a critical amount (4). One can understand localized modes easily by using the analogy with a filter. The crystal may be looked upon as a filter with pass bands consisting of the allowed frequencies of the host crystal. If the defect has a characteristic vibration which is not in the pass band, then the disturbance will 
not propagate through the crystal but will exist at the impurity site as a spatially localized mode.

If the frequency of a normal mode of the perturbed crystal lies in the range of frequencies of the normal modes of the host crystal, then such mode of the perturbed crystal will have the character of ordinary wave-like band modes many atomic distances from the defect. Near the defect the amplitude will be enhanced or attenuated depending on the nature of the changes of the mass and the force constants. Those modes with the enhancement of the amplitude near the defect are called resonance modes.

It should be noted that without knowledge of the force constant changes and the mass change one can not predict the occurrence of localized or resonance modes. Equation (2.2.12) simply gives the normal modes perturbed by the introduction of the impurity; in this case the nature of these modes must be investigated experimentally. 


\section{CHAPTER III}

\section{AN HCP CRYSTAL WITH A DEFECT}

Before making use of the formulation developed in the first chapter, wo discuss the symmetry properties of an HCP crystal with a substitutional defect and obtain the normal modes perturbed by the introduction of the impurity with their degeneracies. Then we select the symmetry adapted linear combinations of the displacement amplitudes (SALC's) for each irreducible representation of the point group $D_{3 h}$ which is the symmetry group of the cluster. This is the starting point for obtaining the solutions of Eq. (2.2.12). The notation and the group theoretical techniques used in this chapter can be found in any standard textbook on group theory $(19-21)$.

\section{DETERMINATION OF NORMAL MODES}

In order to determine the symmetries of the normal modes which are perturbed by the introduction of the impurity, we consider the cluster formed by the impurity atom and its nearest neighbours and develop a representation of the point group of the cluster. In earlier chapter we referred to this as the impurity cluster. The term "quasi-molecule" is also used for the cluster. A crystal with one impurity is a highly idealized picture. A real crystal has many such impurity clusters, separated by several atomic distances so that they can be treated independent of each other. 
Figure I shows the impurity cluster in an HCP crystal. The impurity atom is taken as the origin. The site indices $\{1 k\}$ used in the first chapter take values 0 to 12 . The system of axes is such that the projections of the atoms 8 and 11 on the $y z$ plane lie on the $z$ axis (Figure 2): This cluster has $D_{3 h}$ symmetry. The symmetry operations of this group are listed in Table I and they are indicated in Figure 3.

We make use of the 39 cartesian displacement coordinates $\left\{u_{\alpha}(n)\right\}$ of the atoms of the impurity cluster as the basis for the total representation. $\Gamma_{\text {HCP }}$ of the point group $D_{3 h}$. The site index $(n)$ replaces $(1 x)$ used in Chapter II and takes values 0 to 12 . If $U$ denotes the column vector formed by the Cartesian displacement coordinates $\left\{u_{\alpha}(n)\right\}$ and $U^{\prime}$ denotes the same vector after the crystal has been subjected to a symmetry operation $S$ of the point group $D_{3 h}$, then $\Gamma_{H C p}(S)$ defined below is the representation of $\mathrm{S}$ in the 39 dimensional basis:

$$
U^{*}=\Gamma_{\text {HCP }}(S) U
$$

Instead of the matrix form of $\Gamma_{\text {HCP }}(S)$ we are interested in the character $\chi_{\text {HCp }}(S)$ which is important in group theoretical techniques. The atoms which remain unshifted under the operation $S$ contribute to the diagonal terms of $\Gamma_{\text {HCP }}(S)$ and hence to the character $\chi_{H C P}(S)$. Table II summarizes the work done for determining the character system of the total representation. The total representation can be decomposed into the irreducible representations of the point group $D_{3 h}$ by using the decomposition formula:

$$
a_{j}=(1 / h) \sum_{s} X^{(j)}(s) * \chi_{H(p)}(s)
$$




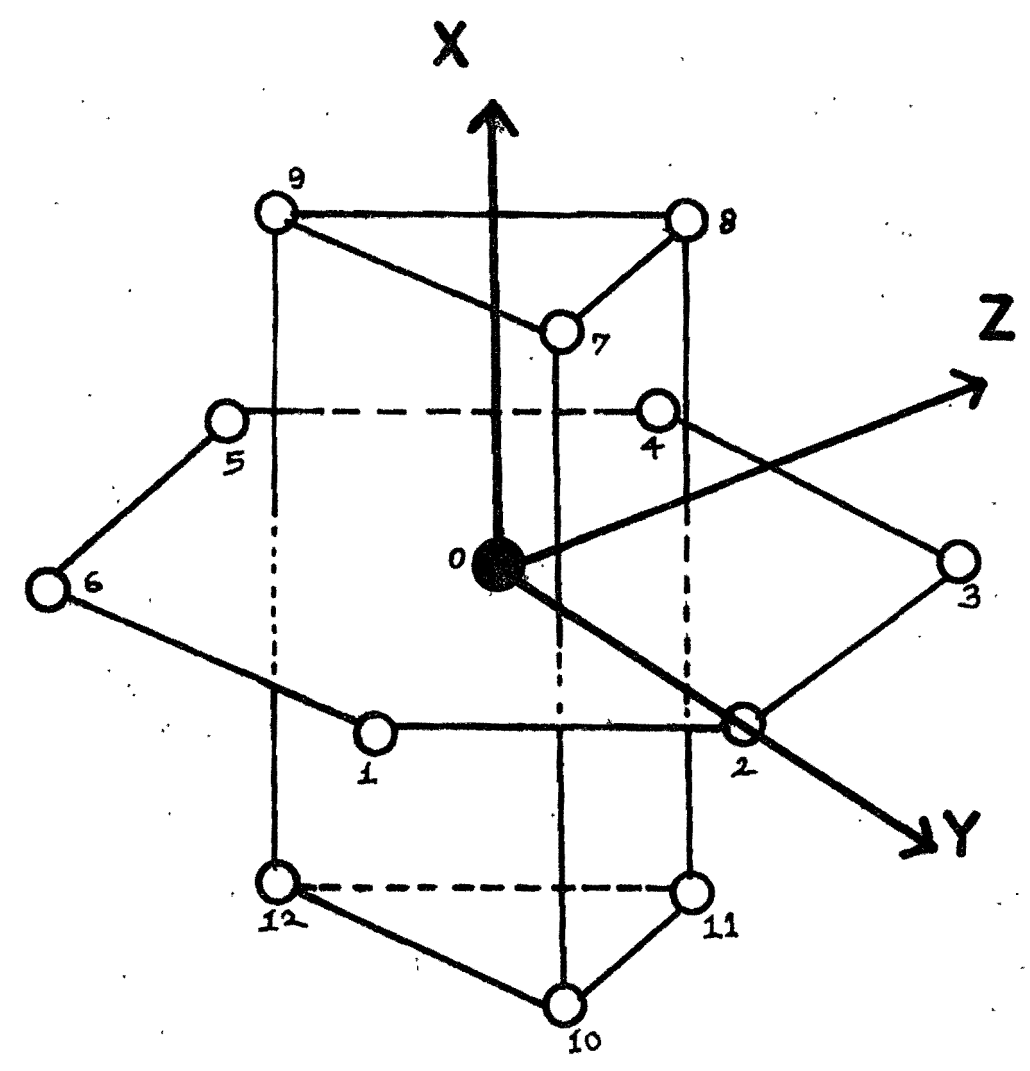

Impurity atom
Host crystal atom

Figure 1. Impurity cluster in an hop crystal. 


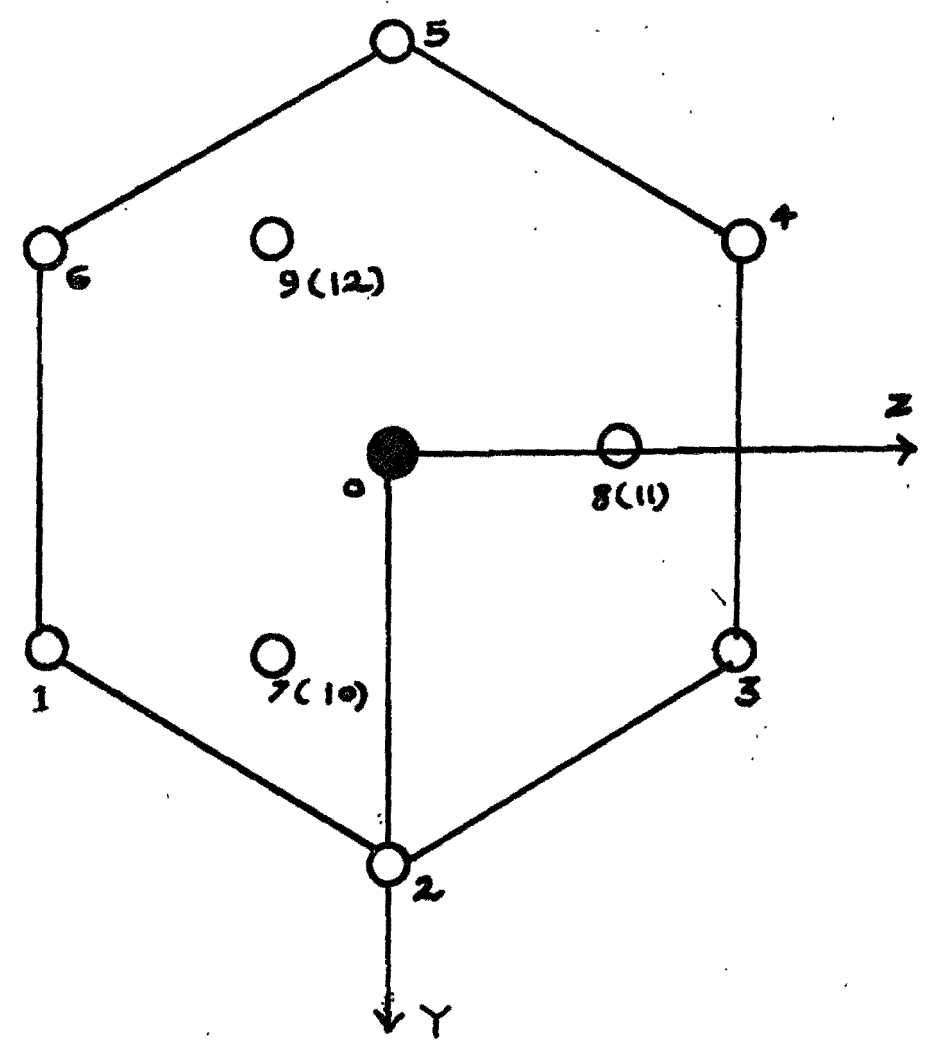

Figure 2. Impurity cluster viewed along the main symmetry axis. 
TABLE I

SYMMETRY OPERATIONS OF THE POINT GROUP $\mathrm{D}_{3 \mathrm{~h}}$

\begin{tabular}{|c|c|c|}
\hline Number & Operation & Description \\
\hline 1 & $E$ & Identity \\
\hline 2,3 & $\mathrm{C}_{3} ; \mathrm{C}_{3}^{-1}$ & Rotations by $2 \pi / 3$ about the $z$ axis; \\
\hline 4 & $\mathrm{C}_{2}^{\prime \prime}(1)$ & Rotation by $\pi$ about the $y$ axis; \\
\hline 5 & $\mathrm{C}_{2}^{\prime \prime}(2)$ & $\begin{array}{l}\text { Rotation by } \pi \text { about the axis deduced from the } \\
\text { y axis by } C_{3} \text {; }\end{array}$ \\
\hline 6 & $\mathrm{CH}_{2}(3)$ & $\begin{array}{l}\text { Rotation by } \pi \text { about the axis deduced from the } \\
\text { y axis by } \mathrm{C}_{3}^{-1} \text {; }\end{array}$ \\
\hline 7 & $\sigma_{h}$ & Reflection in the yz plane; \\
\hline 8,9 & $s_{3}, s_{3}^{-1}$ & $\mathrm{C}_{3}$ and $\mathrm{C}_{3}^{-1}$ followed by $\sigma_{h}$ respectively; \\
\hline 10 & $\sigma_{0}(1)$ & Reflection in the $z x$ plane; \\
\hline 11 & $\sigma_{0}(2)$ & $\begin{array}{l}\text { Reflection in the plane deduced from the } \mathrm{zx} \\
\text { plane by } \mathrm{C}_{3} \text {; }\end{array}$ \\
\hline 12 & $\sigma_{0}(3)$ & $\begin{array}{l}\text { Reflection in the plane deduced from the } \mathrm{zx} \\
\text { plane by } \mathrm{C}_{3}^{-1} \text {. }\end{array}$ \\
\hline
\end{tabular}




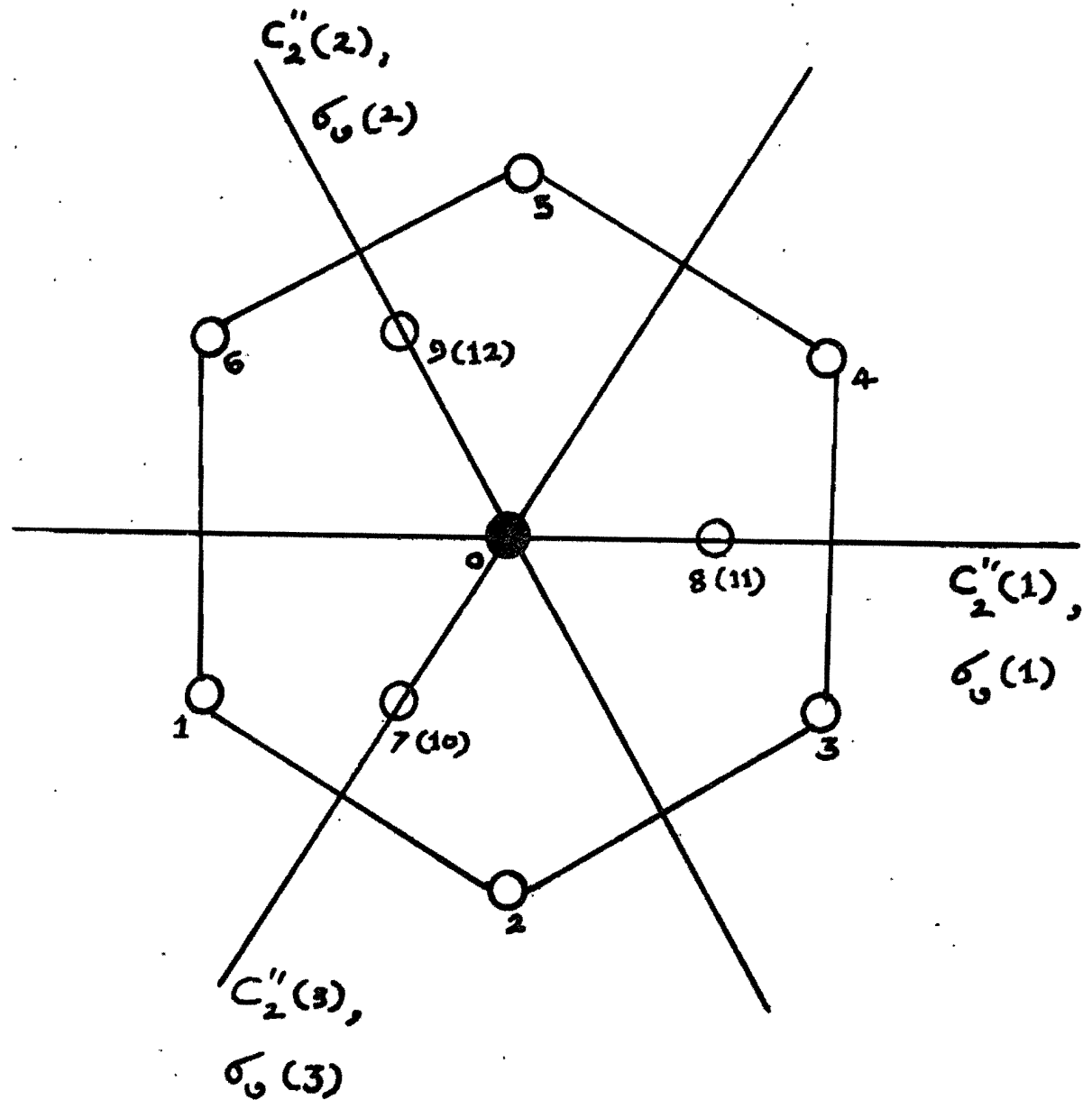

Figure 3. Symmetry operations of the point group $D_{3 h}$. 
TABLE II

DETERMTNATION OF $\mathrm{HCP}_{\mathrm{P}}(\mathrm{R})$

\begin{tabular}{|c|c|c|c|c|c|}
\hline Class & $\begin{array}{l}\text { Symmetry } \\
\text { Operation } \\
R\end{array}$ & $\begin{array}{l}\text { Atom Sites } \\
\text { invariant } \\
\text { under } R\end{array}$ & $\begin{array}{l}\text { Atom Sites varia } \\
\text { initial site }\end{array}$ & $\begin{array}{l}\text { ant under } R \\
\text { Final site after } R\end{array}$ & $\begin{array}{l}\text { Character } \\
\operatorname{HCP}(\mathrm{R})\end{array}$ \\
\hline & $E$ & $\begin{array}{l}\text { All atom } \\
\text { sites invar- } \\
\text { lant }\end{array}$ & - & - & 39 \\
\hline & $\mathrm{C}_{3}$ & 0 & $\begin{array}{l}1,2,3,4,5,6 \\
7,8,9,10,11,12\end{array}$ & $\begin{array}{l}3,4,5,6,1,2 \\
8,9,7,11,12,10\end{array}$ & 0 \\
\hline & $\mathrm{C}_{3}^{-1}$ & 0 & $\begin{array}{l}1,2,3,4,5,6 \\
7,8,9,10,11,12\end{array}$ & $\begin{array}{l}5,6,1,2,3,4 \\
9,7,8,12,10,11\end{array}$ & 0 \\
\hline & $\mathrm{C}_{2}^{\prime \prime}(1)$ & 0 & $\begin{array}{l}1,2,3,4,5,6 \\
7,8,9,10,11,12\end{array}$ & $\begin{array}{l}6,5,4,3,2,1 \\
12,11,10,9,8,7\end{array}$ & -1 \\
\hline & $\mathrm{C}_{2}^{\prime \prime}(2)$ & 0 & $\begin{array}{l}1,2,3,4,5,6 \\
7,8,9,10,11,12\end{array}$ & $\begin{array}{l}4,3,2,1,6,5 \\
11,10,12,8,7,9\end{array}$ & -1 \\
\hline & $C_{2}^{\prime \prime}(3)$ & 0 & $\begin{array}{l}1,2,3,4,5,6 \\
7,8,9,10 ; 11,12\end{array}$ & $\begin{array}{l}2,1,6,5,4,3 \\
10,12,11,7,9,8\end{array}$ & -1 \\
\hline & $\sigma_{h}$ & $0,1,2,3,4,5,6$ & $\overline{7}, 8,9,10,11,12$ & $\overline{10}, 11,12,7,8,12$ & 7 \\
\hline & $\mathrm{S}_{3}$ & 0 & $\begin{array}{l}1,2,3,4,5,6 \\
7,8,9,10,11,12\end{array}$ & $\begin{array}{l}3,4,5,6,1,2 \\
11,12,10,8,9,7\end{array}$ & -2 \\
\hline
\end{tabular}


TABLE II (continued)

\begin{tabular}{|c|c|c|c|c|c|}
\hline Class & $\begin{array}{c}\text { Symmetry } \\
\text { Operation } \\
\mathrm{R}\end{array}$ & $\begin{array}{l}\text { Atom Sites } \\
\text { invariant } \\
\text { Under } \mathrm{R}\end{array}$ & $\begin{array}{l}\text { Atom Sites variant under } \mathrm{R} \\
\text { initial site }\end{array}$ & Final site after $\mathrm{R}$ & $\begin{array}{c}\text { Character } \\
\text { HCP }\end{array}$ \\
\hline $\begin{array}{c}C_{5} \\
\text { (cont.) }\end{array}$ & $\mathrm{S}_{3}^{-1}$ & 0 & $\begin{array}{l}1,2,3,4,5,6 \\
7,8,9,10,11,12\end{array}$ & $\begin{array}{l}5,6,1,2,3,4 \\
12,10,11,9,7,8\end{array}$ & -2 \\
\hline$C_{6}$ & $(1)$ & $0,8,11$ & $\begin{array}{l}1,2,3,4,5,6 \\
7,9,10,12\end{array}$ & $\begin{array}{l}6,5,4,3,2,1, \\
9,7,12,10\end{array}$ & 3 \\
\hline$C_{6}$ & $(2)$ & $0,9,12$ & $\begin{array}{l}1,2,3,4,5,6 \\
7,8,10,12\end{array}$ & $\begin{array}{l}4,3,2,1,6,5 \\
8,7,11,10\end{array}$ & 3 \\
\hline$C_{6}$ & (3) & $0,7,10$ & $\begin{array}{l}1,2,3,4,5,6 \\
8,9,11,12\end{array}$ & $\begin{array}{l}2,1,6,5,4,3 \\
9,8,12,11\end{array}$ & 3 \\
\hline
\end{tabular}


where $a_{j}$ is the number of times the irreducible representation $\Gamma^{(j)}$ appears in the reducible representation $\Gamma_{H C p}, h$ is the number of elements in the group, $\chi_{\mathrm{HC}}(\mathrm{S})$ is the character of the total representation of the operation $S$, and $\ddot{X}^{(j)}(S)$ is the character of the $j^{\text {th }}$ irreducible representation of the same operation S. Table III gives the character system for the point group $D_{3 h}$ in which the last column gives the number $a_{j}$. Now the decomposition of $\Gamma_{\mathrm{H} C \mathrm{p}}$ into the irreducible representations of the point group $D_{3 h}$ can be written as:

$\Gamma_{H C P}=4 A_{1}^{\prime}+3 A_{2}^{\prime}+2 A_{1}^{\prime \prime}+4 A_{2}^{\prime \prime}+8 E^{\prime}+5 E^{\prime \prime}$

In the above decomposition $A^{\prime}$ s correspond to nondegenerate normal modes of the cluster and E's which are two dimensional irreducible representations correspond to doubly degenerate normal modes of the cluster. In molecular vibrations, the conservation of the linear momentum and the angular momentum of the molecule requires that the modes which represent rigid body translation or rigid body rotation be removed from the decomposition series. The impurity cluster which we are considering is emobdied in a crystal and hence its linear momentum and the angular momentum need not be conserved. Hence as the part of a vibration of the crystal this cluster can have normal modes of vibration which may appear to be rigid body translation or rigid body rotation. For this reason all the normal modes in Eq. (3.1.3) will be retained.

\section{THE DEFECT MOTION}

In the study of the dynamics of crystals with substitutional impurities, particularly in MU ssbauer effect, the motion of the impurity atom 
TABLE III

CHARACTER SYSTEM FOR THE POINT GROUP $D_{3 h}$

\begin{tabular}{|l|c|c|c|c|c|c|c|}
\hline Class & $E$ & $\sigma_{h}$ & $2 \mathrm{C}_{3}$ & $2 \mathrm{~S}_{3}$ & $3 \mathrm{C}_{2}^{\prime \prime}$ & $3 \sigma_{5}$ & $\mathrm{a}_{j}$ \\
\hline$\Gamma_{1}^{\prime(j)}$ & 1 & 1 & 1 & 1 & 1 & 1 & 4 \\
$\mathrm{~A}_{2}^{\prime}$ & 1 & 1 & 1 & 1 & -1 & -1 & 3 \\
$\mathrm{~A}_{1}^{\prime \prime}$ & 1 & -1 & 1 & -1 & 1 & -1 & 2 \\
$\mathrm{~A}_{2}^{\prime \prime}$ & 1 & -1 & 1 & -1 & -1 & 1 & 4 \\
$\mathrm{E}^{\prime}$ & 2 & 2 & -1 & -1 & 0 & 0 & 8 \\
$\mathrm{E}^{\prime \prime}$ & 2 & -2 & -1 & 1 & 0 & 0 & 5 \\
\hline$\Gamma_{\mathrm{HCP}}$ & 39 & 7 & 0 & -2 & -1 & 3 & \\
\hline
\end{tabular}


is very important. For identifying the modes in which the defect atom participates, we use the projection operator $\rho^{(j)}$ for the $j^{\text {th }}$ irreducible representation as:

$$
\rho^{(j)} \equiv\left[1_{j} / h\right] \sum_{R} \chi^{(j)}(R) P_{R}
$$

where $l_{j}$ is the dimensionality of the $j^{\text {th }}$ irreducible representation, $\left\{\chi^{(j)}(R)\right\}$ is the character system of the $j^{\text {th }}$ irreducible representation. $\mathrm{P}_{\mathrm{R}}$ is the active symmetry operator* of the operation $\mathrm{R}$. From an arbitrary function $\psi$ the operator $\boldsymbol{P}^{(j)}$ projects that part which transforms according to the $j^{\text {th }}$ irreducible representation. In other words, from an arbitrary function $\psi, \rho^{(j)}$ projects that part which belongs to the $j^{\text {th }}$ irreducible representation. We set up the projection operators for the irreducible representations of the point group $D_{3 h}$ and operate on the displacement coordinates $u_{x}(0), u_{y}(0)$, and $u_{z}(0)$ of the defect. The results are listed below:

$$
\begin{aligned}
& \mathcal{P}^{(j)} u_{\alpha}(0)=0 \text { for } \alpha=x, y \text { or } z \text {, and } j=A_{1}^{\prime}, A_{2}^{\prime}, A^{\prime \prime}{ }_{1}, \text { or } E^{\prime \prime},(3.2,2 a) \\
& \rho(j) u_{x}(0)=u_{x}(0) \\
& \rho^{(j)} u_{y}(0)=\rho^{(j)} u_{z}(0)=0, \\
& \rho(j)_{u_{x}}(0)=0 \\
& \rho(j) u_{y}(0)=u_{y}(0) \text {, and } \rho^{(j)} u_{z}(0)=u_{z}(0)
\end{aligned}
$$


Equation (3.2.2a) implies that the defect atom does not move in the normal modes $A_{1}^{\prime}, A_{2}^{\prime}, A_{1}^{\prime \prime}$, and $E^{\prime \prime}$. Equations (3.2.2b) to (3.2.2e) indicate that the defect vibrates along $x$ axis in $A_{2}^{\prime r}$ modes and in the $y z$ plane in $E^{\prime}$ modes. Since $A^{\prime \prime} 2$ appears 4 times and $E^{\prime}$ appears 8 times in the decomposition series in Eq. (3.1.3), there are four nondegenerate modes and eight doubly degenerate modes in which the impurity participates.

The solutions of Eq. (2.2.10) should give the normal modes which appear in the decomposition series in Eq. (3.1.3). Since we are interested in the motion of the impurity, we want to solve that part of Eq. (2.2.10) which gives $4 A^{\text {tr }} 2$ modes and $8 E^{\prime}$ modes. It needs block diagonalization of Eq. $(2,2,10)$ :

$$
\left[\begin{array}{c}
u_{A_{2}^{\prime \prime}} \\
u_{E_{11}^{\prime}} \\
u_{E_{22}^{\prime}} \\
u_{R}
\end{array}\right]=\left[\begin{array}{cccc}
g_{A_{2}^{\prime \prime}} & 0 & 0 & 0 \\
0 & \dot{g}_{E_{11}^{\prime}} & 0 & 0 \\
0 & 0 & g_{E_{22}^{\prime}} & 0 \\
0 & 0 & 0 & g_{R}
\end{array}\right]\left[\begin{array}{cccc}
\delta 1_{A^{\prime \prime}} & 0 & 0 & 0 \\
0 & \delta 1_{E_{1.1}^{\prime}} & 0 \\
0 & 0 & \delta 1_{E_{22}^{\prime}} & 0 \\
0 & 0 & 0 & \delta 1_{R}
\end{array}\right]\left[\begin{array}{c}
u_{A^{\prime \prime} 2} \\
u_{E_{11}^{\prime}} \\
u_{E_{22}^{\prime}} \\
u_{R}
\end{array}\right],
$$

where the subscript $A_{2}^{\prime \prime}$ indicates 4-dimensional block, $E_{11}^{\prime}$ and $E^{\prime} 22$ indicate two 8-dimensional blocks, and $\mathrm{R}$ indicates remaining part. From Eq. (3.2.3) we write the equations which are of interest to us:

$$
\begin{aligned}
& u_{A_{2}^{\prime \prime}}=g_{A_{2}^{\prime \prime}} \delta I_{A^{\prime \prime} 2} u_{A_{2}^{\prime \prime}}, \\
& u_{E_{11}^{\prime}}=g_{E_{11}^{\prime}} \delta 1_{E_{11}^{\prime}} u_{E_{11}^{\prime},},
\end{aligned}
$$




$$
u_{E_{22}^{\prime}}=g_{E_{22}^{\prime}} \delta 1_{E_{22}^{\prime}} u_{E_{22}^{\prime}} \text {. }
$$

Equations (3.2.5a) and (3.2.5b) are degenerate hence it is sufficient to consider only one of them.

In the next chapter we discuss the diagonalization procedure which obtains Eqs. (3.2.4) and (3.2.5b) from Eq. (2.2.10). 


\section{DIAGONALIZATION}

For block diagonalizing Eq. $(2.2 .10)$ to the form shown in Eq. (3.2.3), one has to determine the vectors $u_{A_{2}^{\prime \prime}} \cdot u_{E_{1}^{\prime} l^{\prime}}$ and $u_{E^{\prime}}{ }_{22}$. These column vectors have the form:

$$
u_{A_{2}}=\left[\begin{array}{c}
\alpha_{1} \\
\alpha_{2} \\
\alpha_{3} \\
\alpha_{4}
\end{array}\right] \cdot u_{E_{11}^{\prime}}=\left[\begin{array}{c}
\gamma_{1} \\
\gamma_{2} \\
\vdots \\
\vdots \\
\gamma_{8}
\end{array}\right] \quad u_{E_{22}^{\prime}}=\left[\begin{array}{c}
\beta_{1} \\
\beta_{2} \\
\vdots \\
\beta_{8}
\end{array}\right]
$$

The set $\alpha_{1}, \alpha_{2}, \alpha_{3}$, and $\alpha_{4}$ forms a basis for the irreducible representation $A_{2}$. The sets of $\beta^{\prime}$ 's and $\gamma^{\prime}$ 's are the two bases for the two dtmensional representation $E^{\prime}$. For determination of the basis vectors for various irreducible representations, we use the standard procedure of projection operators, which is illustrated in Appendix IV. The basis vectors for all the irreducible representations appearing in the decomposition serl es in Eq. (3.1.3) are given in the same appendix.

The basis vectors for the representation $\mathrm{Al}_{2}$ are: (over) 


$$
\begin{aligned}
& \alpha_{1}= u_{x}(0) \\
& \alpha_{2}=(1 / \sqrt{6}) \sum_{n=1}^{6} u_{x}(n), \\
& \alpha_{3}=(1 / \sqrt{6}) \sum_{n=7}^{12} u_{x}(n), \\
& \alpha_{4}=(1 / \sqrt{2} 4)\left\{\sqrt{\beta}\left[\left(u_{y}(7)-u_{y}(10)\right)-\left(u_{y}(9)-u_{y}(12)\right)\right]\right. \\
&\left.-\left[\left(u_{z}(7)-u_{z}(10)\right)-2\left(u_{z}(8)-u_{z}(11)\right)+\left(u_{z}(9)-u_{z}(12)\right)\right]\right\} .
\end{aligned}
$$

It has been mentioned that the $E^{\prime}$ modes are doubly degenerate. Hence there are two sets of basis vectors for E' modes. Since the two sets are physically equivalent, we shall use the set of $\beta^{\prime}$ s which is:

$$
\begin{aligned}
& \beta_{1}=u_{y}(0), \\
& \beta_{2}=(1 / \sqrt{6}) \sum_{n=1}^{6} u_{y}(n), \\
& \beta_{3}=(1 / \sqrt{6})\left[\left(u_{z}(1)-u_{z}(6)\right)-\left(u_{z}(2)-u_{z}(5)\right)+\left(u_{z}(3)-u_{z}(4)\right)\right],(4.3 c) \\
& \beta_{4}=(1 / \sqrt{10})\left\{\sqrt{3}\left(u_{z}(1)-u_{z}(6)\right)-\left[\left(u_{y}(2)+u_{y}(5)\right)-\left(u_{y}(3)+u_{y}(4)\right)\right]\right\}(4.3 d) \\
& \beta_{5}=(1 / \sqrt{10})\left\{\sqrt{3}\left(u_{y}(1)+u_{y}(6)\right)-\left[\left(u_{z}(2)-u_{z}(5)\right)+\left(u_{z}(3)-u_{z}(4)\right)\right]\right\},(4.3 e)
\end{aligned}
$$




$$
\begin{aligned}
& \beta_{6}=(1 / \sqrt{6}) \sum_{n=7}^{12} u_{y}(n) \\
& \beta_{7}=(1 / \sqrt{10})\left\{\sqrt{3}\left(u_{y}(8)+u_{y}(1)\right)+\left[\left(u_{z}(7)+u_{z}(10)\right)-\left(u_{z}(9)+u_{z}(12)\right]\right\}(4.3 g)\right. \\
& \beta_{8}=(1 / 2) \cdot\left\{\left(u_{x}(7)-u_{x}(10)\right)-\left(u_{x}(9)-u_{x}(12)\right)\right\}
\end{aligned}
$$

Equation $(2.2 .10)$ which is

$$
u_{1}=\dot{g} \delta u_{1}
$$

can be rewritten in a component form as

$$
u_{\alpha}\left(n_{1}\right)=\sum_{\substack{\beta \\ n_{2} n_{3}}} g_{\alpha \beta}\left(n_{1} ; n_{2} ; \omega^{2}\right) \delta_{1}\left(n_{2} ; n_{3} ; \omega^{2}\right) u_{\gamma}\left(n_{3}\right):
$$

We cah substitute from Eq. (4.5) for the displacements $\left\{u_{\alpha}(n)\right\}^{\prime \prime}$ in the basis vectors. After arranging the terms conveniently each basis vector can be expressed as a linear combination of the basis vectors belonging to that set. For example, $\alpha_{1}$ will then be written as

$$
\alpha_{1}=C_{1} \alpha_{1}+C_{2} \alpha_{2}+C_{3} \alpha_{3}+C_{4} \alpha_{4}
$$

and $\beta_{1}$ will then be written as

$$
\beta_{1}=D_{1} \beta_{1}+D_{2} \beta_{2}+\ldots+D_{7} \beta_{7}+D_{8} \beta_{8}
$$

where the coefficients $C_{1}$ to $C_{4}$ and $D_{1}$ to $D_{8}$ are linear combinations of the terms such as $g_{\alpha \beta}\left(n_{1} n_{2}\right) \delta I_{\gamma \delta}\left(n_{3} n_{4}\right)$.

After going through the procedure explained above one can write down the equations for the basis vectors of the $\mathrm{A}^{\prime \prime} 2$ modes: 
37

$$
\begin{aligned}
\alpha_{1}= & \left\{g_{0} \delta l_{x x}(0 ; 0)+6\left[g_{1} \delta l_{x x}(0 ; 1)+g_{2} \delta l_{x x}(0 ; 8)+g_{3} \delta l_{x x}(0 ; 8)\right]\right\} \alpha_{1} \\
& +\sqrt{6}\left\{\left[g_{0}-g_{1}\right] \delta l_{x x}(0 ; 1)\right\} \alpha_{2} \\
& +\sqrt{6}\left\{\left[g_{1}-g_{2}\right] \delta l_{x x}(0 ; 8)-g_{3} \delta l_{x z}(0 ; 8)\right\} \alpha_{3} \\
& +2 \sqrt{6}\left\{\left[g_{0}-g_{2}\right] \delta l_{x z}(0 ; 8)-g_{3} \delta l_{x z}(0 ; 8)\right\} \alpha_{4},
\end{aligned}
$$

(4.8a)

$$
\begin{aligned}
\alpha_{2}= & \sqrt{6}\left\{g_{1} \delta l_{x x}(0 ; 0)+A \delta l_{x x}(0 ; 1)+2 B \delta l_{x x}(0 ; 8)+C \delta l_{x z}(0 ; 8)\right\} \alpha_{1} \\
+ & \left\{\left[6 g_{1}-A\right] \delta l_{x x}(0 ; 1)\right\} \alpha_{2} \\
& +\left\{\left[6 g_{1} \delta l_{x x}(0 ; 8)-2 B \delta l_{x x}(0 ; 8)-C \delta l_{x z}(0 ; 8)\right]\right\} \alpha_{3} \\
& +2\left\{\left[6 \cdot g_{1}-2 B\right] \delta l_{x z}(0,-8)-C \delta l_{z z}(0 ; 8)\right\} \alpha_{4}, \quad(4.8 \mathrm{~b}) \\
\alpha_{3}= & \sqrt{6}\left\{g_{2} \delta l_{x x}(0 ; 0)+2 B \delta l_{x x}(0 ; 1)+D \delta l_{x x}(0 ;-8)+2 g_{4} \delta l_{x z}(0 ; 8)\right\} \alpha_{1} \\
+ & \left\{\left(6 g_{2}-2 B\right) \delta l_{x x}(0 ; 1)\right\} \alpha_{2} \\
& +\left\{\left(6 g_{2}-D\right) \delta l_{x x}(0 ; 8)-2 g_{4} \delta l_{x z}(0 ; 8)\right\} \alpha_{3} \\
& +\left\{\left(6 g_{2}-D\right) \delta l_{x z}(0 ; 8)-2 g_{4} \delta l_{2 z}(0 ; 8)\right\} \alpha_{4}, \quad(4.8 \mathrm{c})
\end{aligned}
$$




$$
\begin{aligned}
\alpha_{4}= & (\sqrt{6} / 2)\left\{g_{3} \delta l_{x x}(0 ; 0)+C \delta l_{x x}(0 ; 1)+2 g_{4} \delta l_{x x}(0 ; 8)+F \delta l_{x z}(0 ; 8)\right\} \alpha_{1} \\
& +(1 / 2)\left\{\left[6 g_{3}-C\right] \delta l_{x x}(0 ; 1)\right\} \alpha_{2} \\
& +(1 / 2)\left\{\left[6 g_{3}-2 g_{4}\right] \delta l_{x x}(0 ; 8)\right\} \alpha_{3} \\
& +\left\{\left[6 g_{3}-2 g_{4}\right] \delta l_{x z}(0 ; 8)-F \delta l_{z 2}(0 ; 8)\right\},
\end{aligned}
$$

38

where

$$
\begin{aligned}
g_{0}= & g_{x x}(0 ; 0), \quad g_{1}=g_{x x}(0 ; 1) \\
g_{1}= & g_{x x}(0 ; 8), \quad g_{3}=g_{x z}(0 ; 8), g_{4}=g_{x z}(8 ; 10), \quad(4.9) \\
A= & g_{x x}(0 ; 0)+2 g_{x x}(0 ; 1)+g_{x x}(1 ; 3)+g_{x x}(1 ; 4)+g_{x x}(1 ; 5)(4.10 a) \\
B= & g_{x x}(0 ; 8)+g_{x x}(1 ; 8)+g_{x x}(1 ; 9), \\
C= & 2 g_{x z}(1 ; 8)+2 g_{x z}(1 ; 9)-g_{x z}(0 ; 8), \\
D= & 2 g_{x x}(8 ; 7)+g_{x x}(8 ; 8)+2 g_{x x}(8 ; 10)+g_{x x}(8,11), \\
F= & {\left[g_{y y}(8 ; 8)-g_{y y}(8 ; 11)\right]-\left[g_{z z}(8 ; 7)-\sqrt{3} g_{z y}(8 ; 7)\right] } \\
& +\left[g_{z z}(8 ; 10)-\sqrt{3} g_{z y}(8 ; 10)\right] .
\end{aligned}
$$


The preceding equations can be written in a matrix form:

$$
u_{A_{2}^{\prime \prime}}=g_{A_{2}^{\prime \prime}} \delta l_{A_{2}^{\prime \prime}} u_{A_{2}^{\prime \prime}}
$$

which is Eq. (3.2.4). The column vector $U_{A_{2}^{\prime \prime}}$ is defined in Eq. (4.1) and $g_{A_{2}^{\prime \prime}}$ and $\delta l_{A_{2}^{\prime \prime}}$ are given by

$$
g_{A_{2}^{n}}=\left[\begin{array}{cccc}
g_{0} & \sqrt{6} g_{1} & \sqrt{6} g_{2} & (\sqrt{6} / 2) g_{3} \\
\sqrt{6} g_{1} & A & 2 B & (1 / 2) C \\
\sqrt{6} g_{2} & 2 B & D & g_{4} \\
(\sqrt{6} / 2) g_{3} & (1 / 2) C & g_{4} & (1 / 4) F
\end{array}\right],
$$

$$
\delta l_{A_{2} \prime}=\left[\begin{array}{cccc}
\delta l_{x x}(0 ; 0) & \sqrt{6} \delta l_{x x}(0 ; 1) & \sqrt{6} \delta l_{x x}(0 ; 8) & 2 \sqrt{6} \delta l_{x z}(0 ; 8) \\
\sqrt{6} \delta l_{x x}(0 ; 1) & -\delta l_{x x}(0 ; 1) & 0 & 0 \\
\sqrt{6} \delta l_{x x}(0,8) & 0 & -\delta l_{x x}(0 ; 8) & -2 \delta l_{x z}(0,8) \\
2 \sqrt{6} \delta l_{x z}(0 ; 8) & 0 & -2 \delta l_{x z}(0 ; 8) & -4 \delta l_{z z}(0,8)
\end{array}\right],
$$


We can treat the set of basis vectors given in Eq. (4.3) in the same way and obtain eight equations similar to Eq. (4.7). From these eight equations we can write down the matrix product $g_{E_{22}^{\prime}} \sigma_{1} E_{22}^{\prime}$. We can choose symmetric matrices $g_{E_{22}^{\prime}}$ and $\delta I_{E_{22}^{\prime}}$ by trial and error approach which give the correct $g_{E_{22}^{\prime}} \delta l_{E_{22}^{\prime}}$ product. Since this calculation is Voch tedious, details can not be given here. The elements of matrices

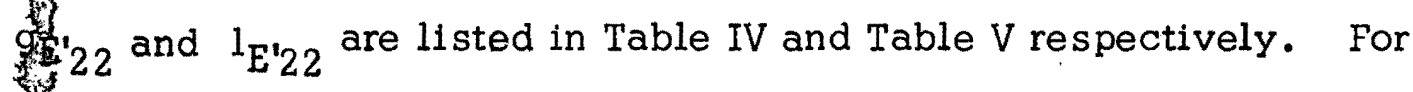
Convenience we have indicated the site indices by the superscripts, for example: $g_{\alpha \beta}\left(n_{1}, n_{2}, \omega^{2}\right)$ is written as $g_{\alpha \beta}^{n_{1} n_{2}}$ where $n_{1}, n_{2}=0,1, \ldots$ 12. 
TABLE IV

ELEMENTS OF THE MATRIX $g_{E_{22}^{\prime}}$

\begin{tabular}{|c|c|}
\hline $\begin{array}{l}\text { Row } \\
\text { and } \\
\text { Column } \\
\text { Number }\end{array}$ & Matrix Element \\
\hline$\left(\begin{array}{ll}1 & 1\end{array}\right)$ & $g_{y y}^{00}$ \\
\hline$\left(\begin{array}{ll}1 & 2\end{array}\right)$ & $(\sqrt{6} / 2)\left(9_{y y}^{01}+g_{z z}^{01}\right)$ \\
\hline$\left(\begin{array}{ll}1 & 3\end{array}\right)$ & $\sqrt{6}\left[9_{y z}^{01}-(\sqrt{3} / 2)\left(9_{y y}^{01}-g_{z z}^{01}\right)\right]$ \\
\hline$\left(\begin{array}{ll}1 & 4\end{array}\right)$ & $(2 \sqrt{3 / 10})\left[g_{y z}^{01}+(\sqrt{3} / 2)\left(g_{y y}^{01}-g_{z z}^{01}\right)\right]$ \\
\hline$\left(\begin{array}{ll}1 & 5\end{array}\right)$ & $(3 / 10)^{1 / 2}\left(39_{y y}^{01}-g_{z z}^{01}\right)$ \\
\hline$\left(\begin{array}{ll}1 & 6\end{array}\right)$ & $(\sqrt{6} / 2)\left(9_{y y}^{08}+g_{z z}^{0 \theta}\right)$ \\
\hline$\left(\begin{array}{ll}1 & 7\end{array}\right)$ & $(\sqrt{3 / 10})\left(399_{y y}^{08}-9_{z z}^{08}\right)$ \\
\hline$\left(\begin{array}{ll}1 & 8\end{array}\right)$ & $\sqrt{3} 9_{2 x}^{08}$ \\
\hline$\left(\begin{array}{ll}2 & 2\end{array}\right)$ & $g_{y y}^{00}+\left(g_{y y}^{01}+g_{z z}^{01}\right)+\left(g_{y y}^{13}+g_{z z}^{13}\right)+\frac{1}{2}\left(g_{y y}^{14}+g_{z z}^{14}\right)$. \\
\hline$\left(\begin{array}{ll}2 & 3\end{array}\right)$ & $\sqrt{3}\left(g_{y y}^{01}-g_{z z}^{01}\right)-2 g_{y z}^{01}+(\sqrt{3} / 2)\left(g_{y y}^{14}-g_{z z}^{14}\right)-g_{y z}^{14}$ \\
\hline$(24)$ & $(1 / \sqrt{5})\left[-2 g_{y z}^{01}-g_{y z}^{14}+(3 \sqrt{3} / 2)\left(g_{y y}^{14}-g_{z z}^{14}\right)+(\sqrt{3} / 2)\left(g_{y y}^{13}-g_{z z}^{13}\right)\right]$ \\
\hline$(25)$ & $(1 / \sqrt{5})\left[g_{y y}^{00}+2 g_{z z}^{01}+(1 / 2)\left(3 g_{y y}^{13}+g_{z z}^{13}+3 g_{y y}^{14}-g_{z z}^{14}\right)\right]$ \\
\hline$(26)$ & {$\left[g_{y y}^{08}+g_{z z}^{08}+g_{y y}^{18}+g_{z z}^{18}+g_{y y}^{18}+g_{z z}^{19}\right]$} \\
\hline$\left(\begin{array}{ll}2 & 7\end{array}\right)$ & $(1 / \sqrt{5})\left[2 g_{z z}^{08}+3 g_{y y}^{18}-g_{z z}^{18}+3 g_{y y}^{19}-g_{z z}^{19}\right]$ \\
\hline
\end{tabular}


42

TABLE IV (continued)

$(28)(-1 / \sqrt{2})\left(9_{z x}^{08}-2 g_{z x}^{18}\right)$

(3 3) $\left[-4 g_{y y}^{00}+(2 / 3)\left(99_{y y}^{01}-5 g_{z z}^{01}\right)-2 \sqrt{3} 9_{y z}^{01}+\left(3 g_{y y}^{13}+g_{z z}^{13}\right)+\right.$ $\left.+\left(-g_{y y}^{14}+4 g_{z z}^{14}+2 \sqrt{3} g_{y z}^{14}\right)\right]$

(3 4) $(1 / \sqrt{5})\left[-4 g_{y y}^{00}+5 g_{y y}^{01}-3 g_{z z}^{01}-2 \sqrt{3} g_{y z}^{01}+(5 / z) g_{y y}^{13}+(3 / 2) g_{z z}^{13}\right.$

$\left.-5 g_{y y}^{14}+3 g_{z z}^{14}+2 \sqrt{3} g_{y z}^{14}\right]$.

(3 5) $\left.(\therefore 1: \sqrt{5})\left[2 \sqrt{3}\left(g_{y y}^{01}-g_{z z}^{01}\right)-2 g_{y y}^{01}+(\sqrt{3} / 2)\left(g_{y y}^{13}-g_{z z}^{13}\right)-g_{y z}^{14}\right]\right)$ )

(3 6) $-\left(g_{y z}^{18}-g_{z y}^{18}\right)$

(3 7) $(1 / \sqrt{5})\left[g_{y z}^{18}+3 g_{z y}^{18}-\sqrt{3}\left(9_{y y}^{08}-g_{z z}^{08}\right)+2 \sqrt{3}\left(g_{y y}^{19}-g_{z z}^{19}\right)\right]$

(3 8) $(1 / \sqrt{2})\left[\sqrt{3} g_{z x}^{08}-2 g_{y x}^{08}\right]$

(4 4) 0

(4 5) $(1 / 5)\left[\sqrt{3}\left(g_{y y}^{01}-3 g_{z z}^{01}\right)-4 g_{y z}^{01}+g_{y z}^{14}+(\sqrt{3} / z)\left(g_{y y}^{14}+3 g_{z z}^{14}\right)\right.$ $\left.+\sqrt{3}\left(9_{y y}^{13}-g_{z z}^{13}\right)\right]$

(4 6) $(1 / \sqrt{5})\left[-2\left(9_{y y}^{19}-g_{z z}^{19}\right)+\left(g_{y z}^{18}+3 g_{z y}^{18}\right)\right]$

(4 7) $(1 / 5)\left[\sqrt{3}\left(9 y y+3 g_{z z}^{08}\right]-\left(g_{y z}^{08}-9 g_{z y}^{08}\right)-2 \sqrt{3}\left(g_{y y}^{19}+g_{z z}^{19}\right)\right]$

(4 8) $(1 / \sqrt{10})\left(3 \sqrt{3} g_{z x}^{08}+2 g_{y x}^{18}\right)$

(5 5) $(1 / s)\left[\left(3 g_{y y}^{01}+\dot{g}_{z z}^{2}\right)-2 \sqrt{3} \dot{g}_{y z}^{1}+5 g_{y y}^{00}-g_{z z}^{13}-(3 / z)\left(g_{y y}^{14}-g_{z z}^{14}\right)\right]$

(5 6) $\left[(1 / \sqrt{3})\left[\left(3 g_{y y}^{08}-g_{z z}^{08}\right)+\left(3 g_{y y}^{18}-g_{z z}^{18}\right)+\left(3 g_{y y}^{19}-g_{z z}^{11}\right)\right]\right.$

(5 7) $\left[(1 / 5)\left[-2 \sqrt{5} 9_{z z}^{08}+\left(9 g_{y y}^{18}+9 z z\right)+\left(39_{y y}^{19}-59_{z z}^{19}\right)\right]\right.$ 
43

TABLE IV (continued)

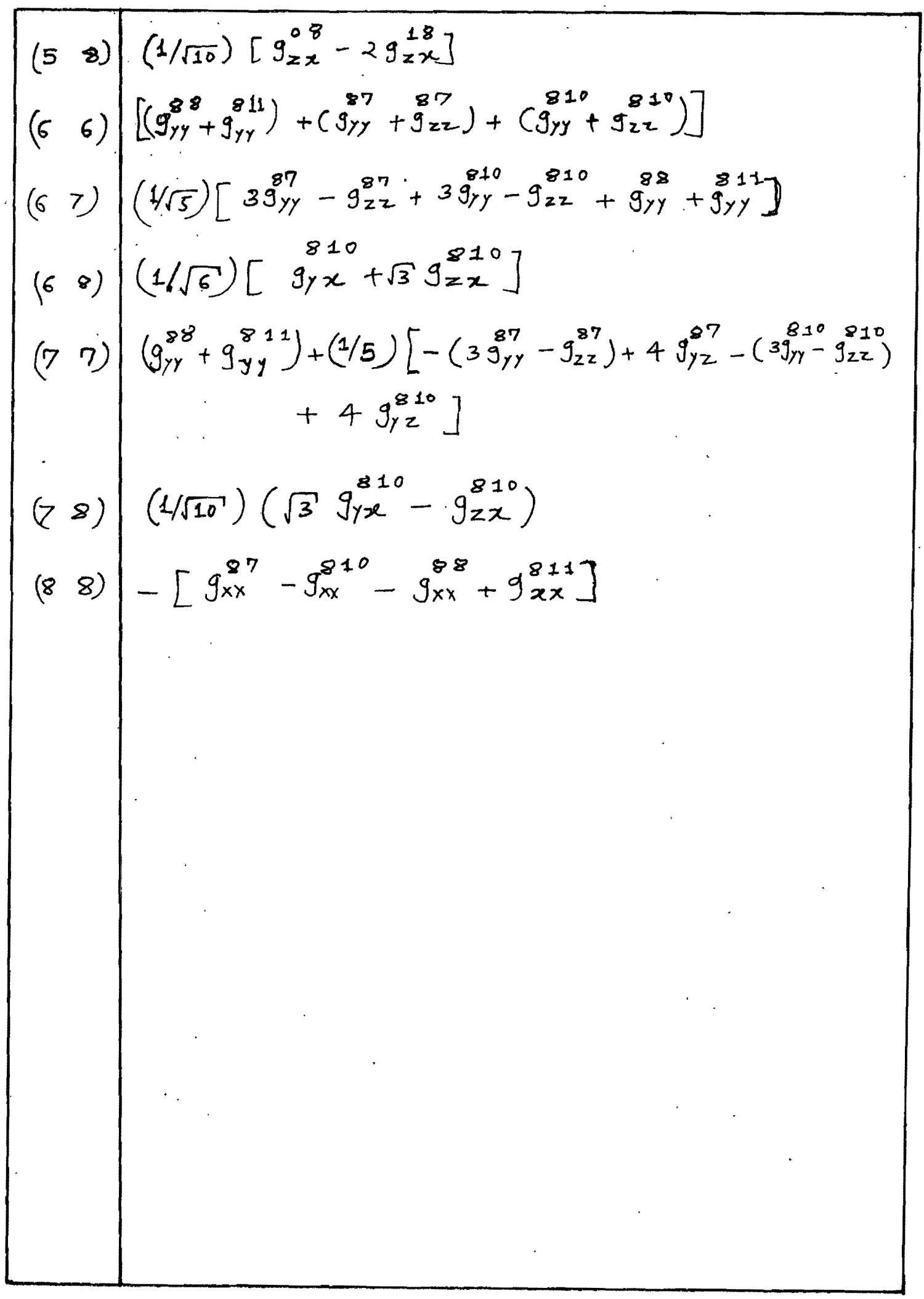


44

TABLE V

ELEMENTS OF THE MATRIX $\delta_{I_{E^{\prime}}}$

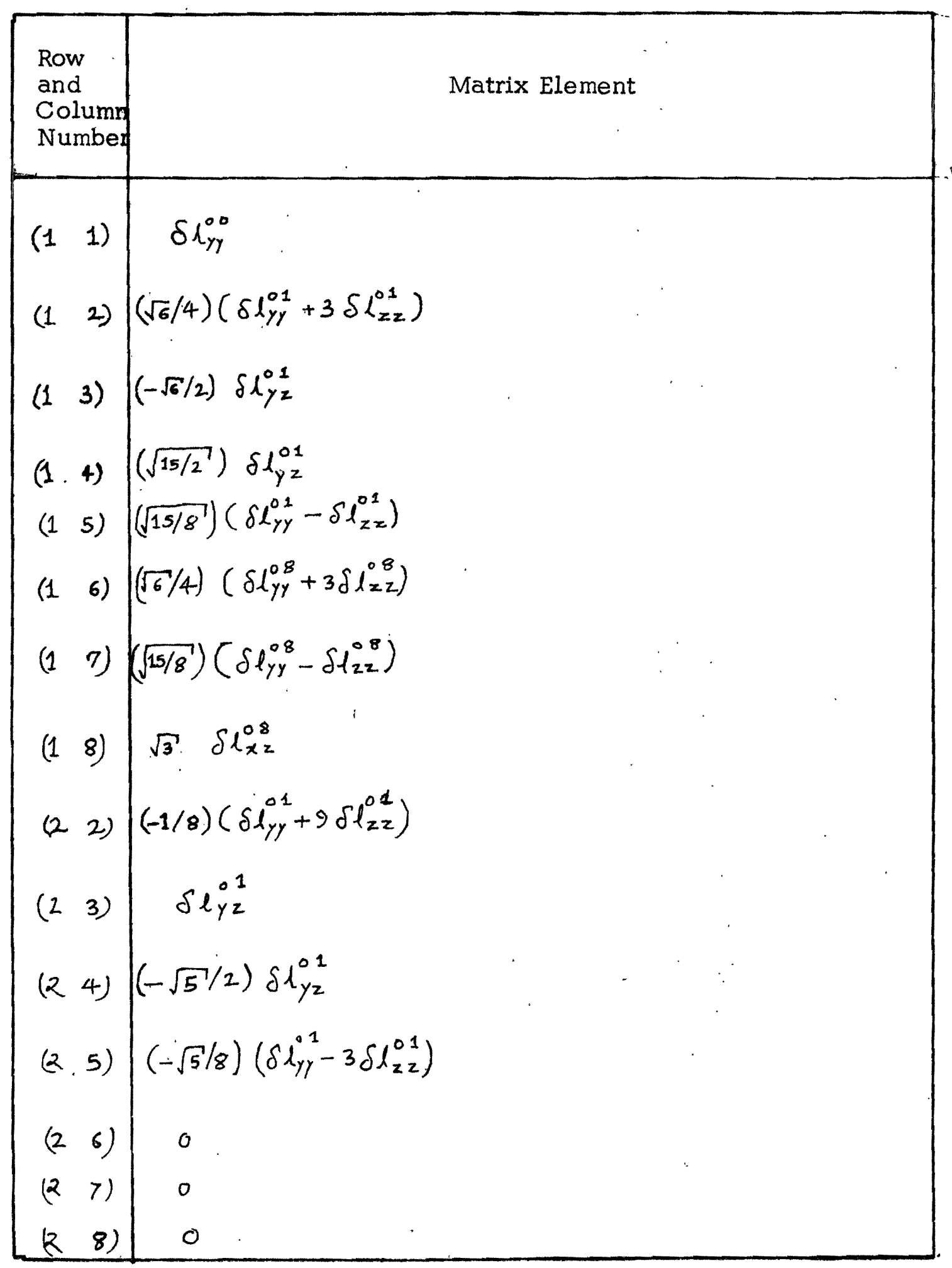


45

TABLE V (continued)

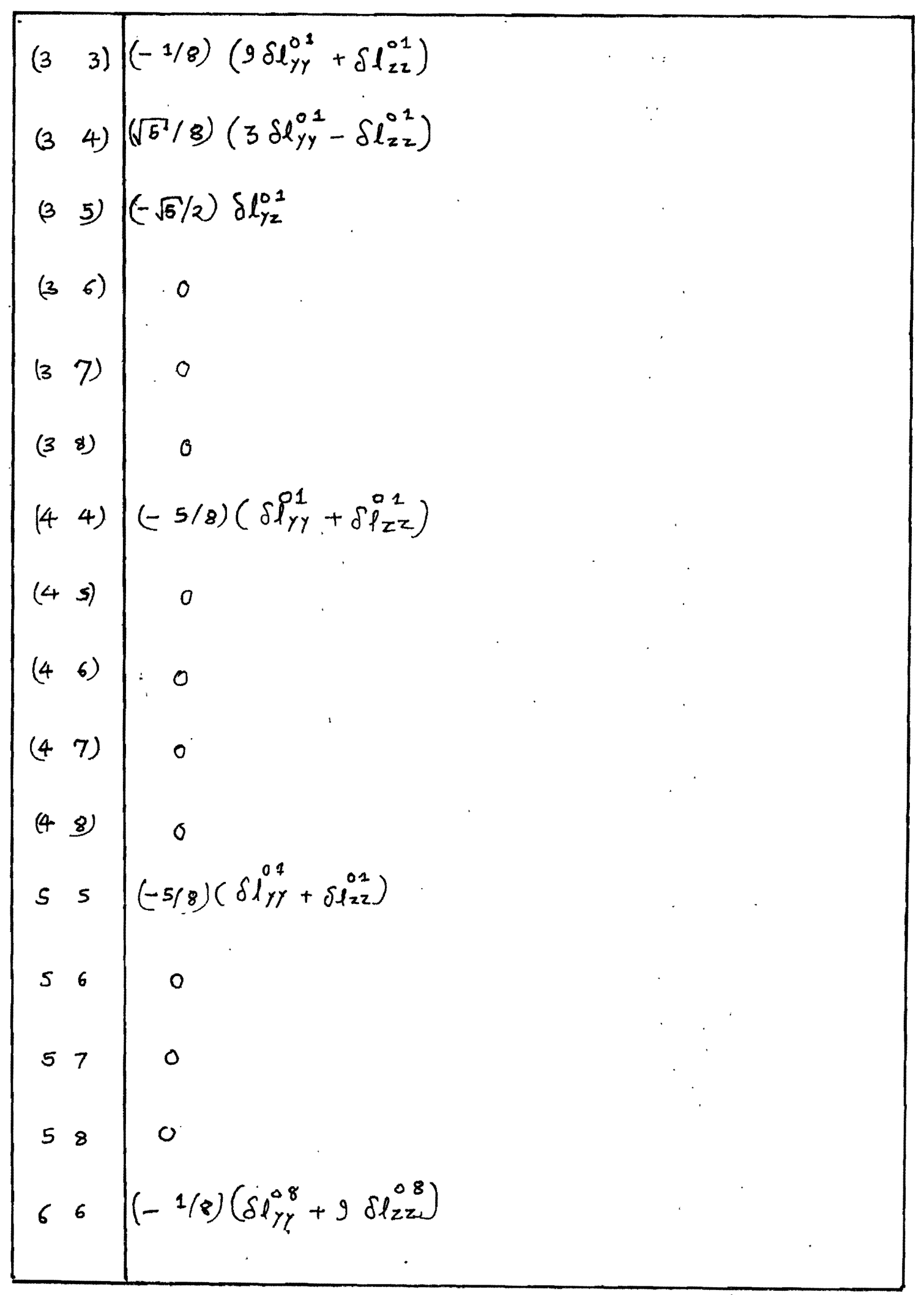


46

TABLE V (continued)

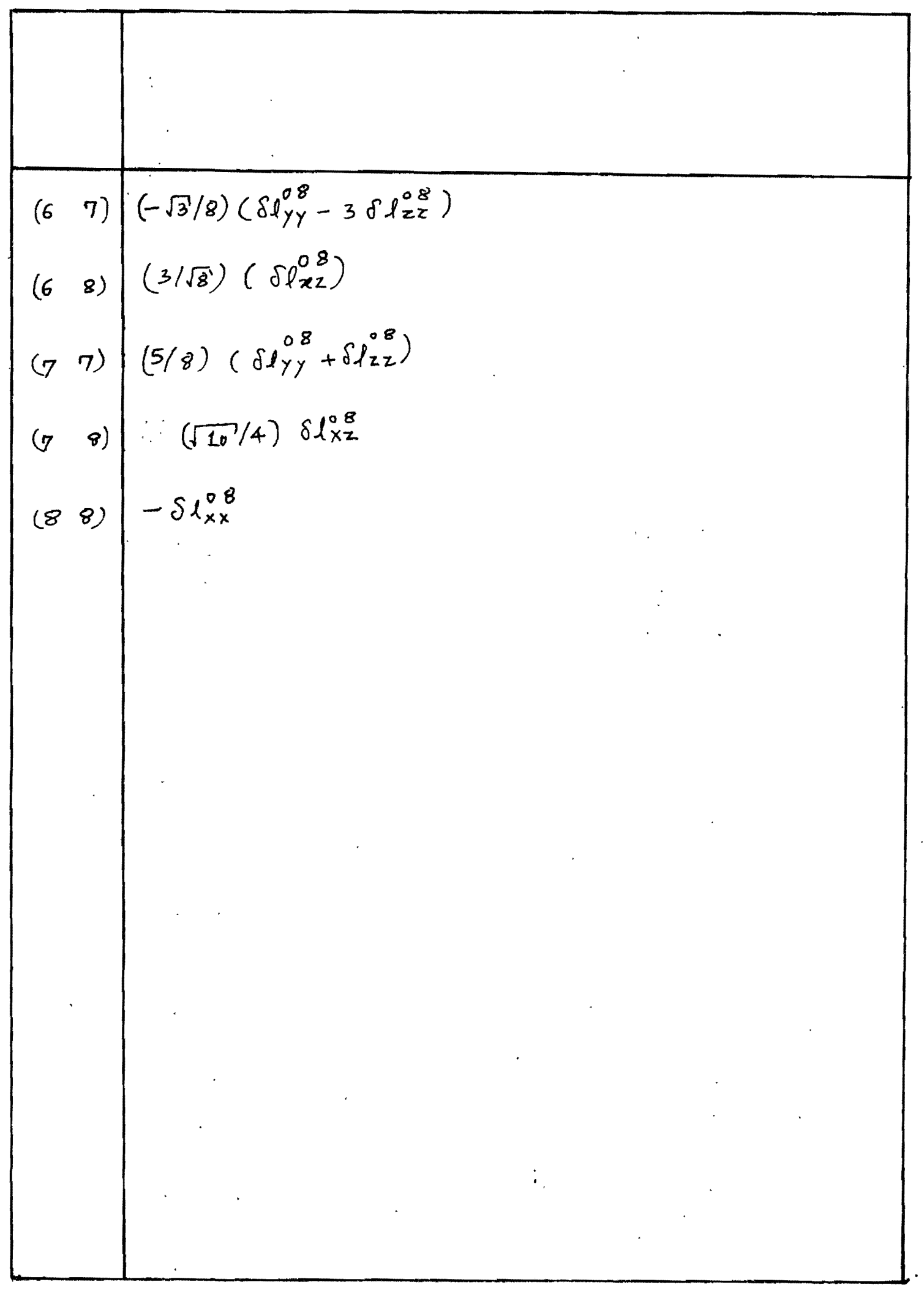


Thus we have obtained Eqs. (3.2.4) and $(3.2 .5 b)$ in explicit forms in which only the linearly independent elements of the matrices $g$ and $\delta 1$ appear. Since neither the elements of $\mathrm{g}$ nor the elements of 61 are known, further calculations will have to be performed numerically. One will have to use a large number of trial values for the elements of the matrices $g$ and $\delta 1$. It should be noted that we did not make any assumption regarding the nature of the atomic interactions while introducing the parameter $\lambda$ describing the force constant change. This allows us to take into account noncentral as well as central interactions in a numerical calculation.

As an illustration of further analytical simplification, in the next chapter we have simplified Eq. $(3.2 .4)$ by assuming central forces. 


\section{CHAPTER V}

\section{THE CASE OF CENTRAL FORCE INTERACTIONS}

In Chapter IV we obtained the equations which must be solved to study the perturbed modes of the imperfect crystal in which the impurity atom participates. The complexity of the equations makes further analytical simplification impossible. In this chapter we shall simplify the equations further by assuming the interatomic interactions to be central. This leads us to some closed analytical results.

\section{ATOMIC FORCE CONSTANTS}

A special case of interatomic potentials is obtained by assuming that the atoms interact pairwise through a potential function which is a function only of the magnitude of their separation. If we denote the potential function for the interaction of an atom of type $*$ with an atom of type $k^{\prime}$ which is at distance $r$ from it by $\phi_{k x^{\prime}}$, then the potential energy of the crystal can be written as:

$$
\Phi=(1 / 2) \sum_{l k} \sum_{l^{\prime} k^{\prime}}^{\prime} \phi_{k k^{\prime}}\left(r\left(l k ; l^{\prime} k^{\prime}\right)\right) .
$$

In this expression $r\left(l_{x} ; l^{\prime} x^{\prime}\right)$ is the instantaneous distance between the atoms $(1 x)$ and $\left(l^{\prime} x^{\prime}\right)$, the prime on the sum indicates that the terms in which $(1 x)=\left(1^{\prime} x^{\prime}\right)$ are to be omitted, while the factor $\mathcal{L} / 2$ corrects for the fact that all the interactions are counted twice in the sum. With the atoms in the crystal displaced by vector displacements $\bar{u}\left(l_{k}\right)$, the expression for $r\left(l_{*} ; I^{\prime} k^{\prime}\right)$ becomes: 
where

$$
\begin{aligned}
& \bar{x}\left(l k_{j} l^{\prime} k^{\prime}\right)=\bar{x}(l k)-\bar{x}\left(l^{\prime} k^{\prime}\right), \\
& \bar{u}\left(l k_{j} l^{\prime} k^{\prime}\right)=\bar{u}(l k)-\bar{u}\left(l^{\prime} k^{\prime}\right) .
\end{aligned}
$$

The potential energy of the crystal can be expanded in the powers of the components $\left\{u_{\alpha}\left(l_{k_{j}} l^{\prime} k^{\prime}\right)\right\}$ as

$$
\begin{aligned}
\Phi= & (1 / 2) \sum_{\substack{l l^{\prime} \\
k k^{\prime}}}^{\prime} \phi_{k k^{\prime}}\left(x\left(l k, l^{\prime} k^{\prime}\right)\right) \\
& +(1 / 2) \sum_{\substack{l l^{\prime} \\
k k^{\prime} \alpha}} \phi_{\alpha}\left(l k ; l^{\prime} k^{\prime}\right) u_{\alpha}\left(l k, l^{\prime} k^{\prime}\right) \\
& +(1 / 4) \sum_{\substack{l l^{\prime} \\
k k_{\alpha \beta}}} \phi_{\alpha \beta}\left(l k ; l^{\prime} k^{\prime}\right) u_{\alpha}\left(l k, l^{\prime} k^{\prime}\right) u_{\beta}\left(l k, l^{\prime} k^{\prime}\right)+\ldots . .
\end{aligned}
$$

The expansion coefficients are given by

$$
\begin{aligned}
& \phi_{\alpha}\left(l k ; l^{\prime} x^{\prime}\right)=\left.\frac{\partial}{\partial x_{\alpha}} \phi_{k k^{\prime}}(r)\right|_{\bar{r}=\bar{x}\left(l x, l^{\prime} x^{\prime}\right)} \\
& =\frac{x_{\alpha}}{r} \phi^{\prime}(r) \mid \bar{r}=\bar{x}\left(l_{k}, l^{\prime} x^{\prime}\right) \text {, } \\
& \phi_{\alpha \beta}\left(l_{k} ; l^{\prime} x^{\prime}\right)=\left.\frac{\partial^{2}}{\partial x_{\alpha} \partial x_{\beta}} \phi_{k k^{\prime}}(r)\right|_{\bar{r}=\bar{x}\left(l_{k}, l^{\prime} k^{\prime}\right)} \\
& \left.=\left\{\frac{x_{\alpha} x_{\beta}}{r}\left[\phi^{\prime \prime}(r)-\phi^{\prime}(r) / r\right]+\frac{\delta_{\alpha \beta}}{r} \phi^{\prime}(r)\right\} \mid \bar{r}=\bar{X}\left(l k_{i} l^{\prime} k^{\prime}\right), 7\right)
\end{aligned}
$$

where the primes denote differentiation with respect to the argument. The first derivative of the potential has to vanish when the atoms are at equilibrium distance since that corresponds to the minimum of the potential energy:

$$
\phi_{\alpha}\left(l_{k} ; l^{\prime} k^{\prime}\right)=0,
$$


which reduces $\mathrm{Eq} .(5 \cdot 1.7)$ to

$$
\phi_{\alpha p}\left(l_{k}, l^{\prime} x^{\prime}\right)=\left.\frac{x_{\alpha} x_{p}}{r^{2}} \phi^{\prime \prime}(r)\right|_{\bar{r}}=\bar{x}\left(l x, l^{\prime} x^{\prime}\right) \text {. }
$$

The second order contribution to the potential in Eq. (5.1.5) can be written as:

$$
\begin{aligned}
\Phi_{2}= & (1 / 4) \sum_{l_{\alpha} k} \sum_{l_{\beta}^{\prime} k^{\prime}}^{\prime} \phi_{\alpha \beta}\left(l k_{i} l^{\prime} k^{\prime}\right)\left[u_{\alpha}(l k) u_{\beta}(l k)-u_{\alpha}\left(l^{\prime} k^{\prime}\right) u_{\beta}\left(l^{\prime} k^{\prime}\right)\right. \\
& \left.-u_{\alpha}\left(l^{\prime} k^{\prime}\right) u_{\beta}(l k)+u_{\alpha}(l k) u_{\beta}\left(l^{\prime} k^{\prime}\right)\right] \\
= & (1 / 2) \sum_{l \times k} \sum_{l^{\prime} k^{\prime} \beta}^{\prime} \phi_{\alpha \beta}\left(l k, l^{\prime} k^{\prime}\right)\left[u_{\alpha}\left(l_{k}\right) u_{\beta}(l k)-u_{\alpha}\left(l_{k}\right) u_{\beta}\left(l^{\prime} k^{\prime}\right)\right](5.1 .10)
\end{aligned}
$$

Again the comparison of Eq. (5.1.10) and Eq. (2.1.4) gives us:

$$
\begin{aligned}
& \Phi_{\alpha \beta}\left(l_{k} l_{k}^{\prime}\right)=-\phi_{\alpha \beta}\left(l_{k}, l_{k}^{\prime}\right) \quad l_{k} \neq l^{\prime} k^{\prime}, \\
& \Phi_{\alpha \beta}\left(l_{k}, l_{k}\right)=\sum_{l^{\prime} k^{\prime}}^{\prime} \phi_{\alpha \beta}\left(l_{k} ; l^{\prime} k^{\prime}\right) .
\end{aligned}
$$

One can see that Eq. (2.3.9) and Eq. (5.1.15) are equivalent.

Equations $(5.1 .9),(5.1 .11)$, and $(5.1 .12)$ imply that

$$
\Phi_{\alpha \beta}\left(l_{x} ; l^{\prime} x^{\prime}\right)=-\left[x_{\alpha} x_{\beta} \phi(r) / r^{2}\right]_{\bar{r}-\bar{x}\left(l x_{j} l^{\prime} x^{\prime}\right)} l^{\prime} f^{\prime} l^{\prime} x^{\prime}(5.1 .13)
$$

and

$$
\Phi_{\alpha \beta}\left(l_{k}, l_{k}\right)=-\sum_{l^{\prime} k^{\prime}}^{\prime} \Phi_{\alpha \beta}\left(l k, l^{\prime} k^{\prime}\right) \text {. }
$$

All the results up to this point in this chapter are valid for a perfect and an imperfect crystal. Using Eq. (5.1.13) in Eq. (2.2.3) we obtain

$$
\delta L_{\alpha \beta}\left(l_{k}, l^{\prime} k^{\prime}\right)=\in M_{k} \omega^{2} \delta_{l l^{\prime}} \delta_{k k^{\prime}} \delta_{\alpha \beta}+\left.\lambda(r) \frac{x_{\alpha} x_{\beta}}{r^{2}} \phi_{p u r e}^{\prime \prime}(r)\right|_{\bar{r}=\bar{x}\left(l_{k}, l_{k^{\prime}}\right),}(5,1.15)
$$

where

$$
\lambda(r)=\left[\phi_{\text {pure }}^{\prime \prime}(r)-\phi_{\text {impure }}^{\prime \prime}(r)\right] / \phi_{\text {pure }}^{\prime \prime}(r) \text {. }
$$

By considering the geometry of the impurity cluster shown in Figure 4 and using the expression given in Eq. (5.1.18) we can write the important $(3 \times 3)$ submatrices of the $\delta \backslash$ matrix as: 
51

$$
\delta l(0 ; 1)=\left[\begin{array}{ccc}
0 & 0 & 0 \\
0 & -(1 / 4) \lambda_{1} \phi^{\prime \prime}(a) & (\sqrt{3} / 4) \lambda_{1} \phi^{\prime \prime}(a) \\
0 & (\sqrt{3} / 4) \lambda_{1} \phi^{\prime \prime}(a) & -(3 / 4) \lambda_{2} \phi^{\prime \prime}(a)
\end{array}\right.
$$

$$
\delta l(0,8)=\left[\begin{array}{ccc}
-\eta_{1}^{2} \lambda_{2} \phi^{\prime \prime}(b) & 0 & -\eta_{1} \eta_{2} \lambda_{2} \phi^{\prime \prime}(b) \\
0 & 0 & 0 \\
-\eta_{1} \eta_{2} \lambda_{2} \phi^{\prime \prime}(b) & 0 & -\eta_{2}^{2} \lambda_{2} \phi^{\prime \prime}(b)
\end{array}\right],
$$

where

$$
\begin{aligned}
& \lambda_{1}=\lambda(a), \quad \lambda_{2} \in(\lambda)(b) \\
& \eta_{1}=c / 2 b, \quad \eta_{2}\left(1-\eta_{1}^{2}\right)^{2 / 2} .
\end{aligned}
$$

The distances $\mathrm{a}, \mathrm{b}$, and $\mathrm{c}$ are shown on Figure 4.

From Eqs. (5.1.17) and (5.1.18) we see that

$$
\begin{aligned}
& \delta l_{x x}(0 ; 1)=0, \\
& \delta l_{z z}(0 ; 1)=3 \delta l_{y y}(0 ; 1), \\
& \delta l_{y z}(0 ; 1)=\delta l_{z y}(0 ; 1)=-\sqrt{3} \delta l_{y y}(0 ; 1), \\
& \delta l_{x z}(0 ; 8)=\delta l_{z x}(0 ; 8)=\left(\eta_{z} / \eta_{1}\right) \delta l_{x x}(0,8), \\
& \delta l_{z z}(0 ; 8)=\left(\eta_{z} / \eta\right) \delta l_{x z}(0 ; 8)=\left(\eta_{z}^{2} / \eta_{1}^{2}\right) \delta l_{x x}(0 ; 8) .
\end{aligned}
$$




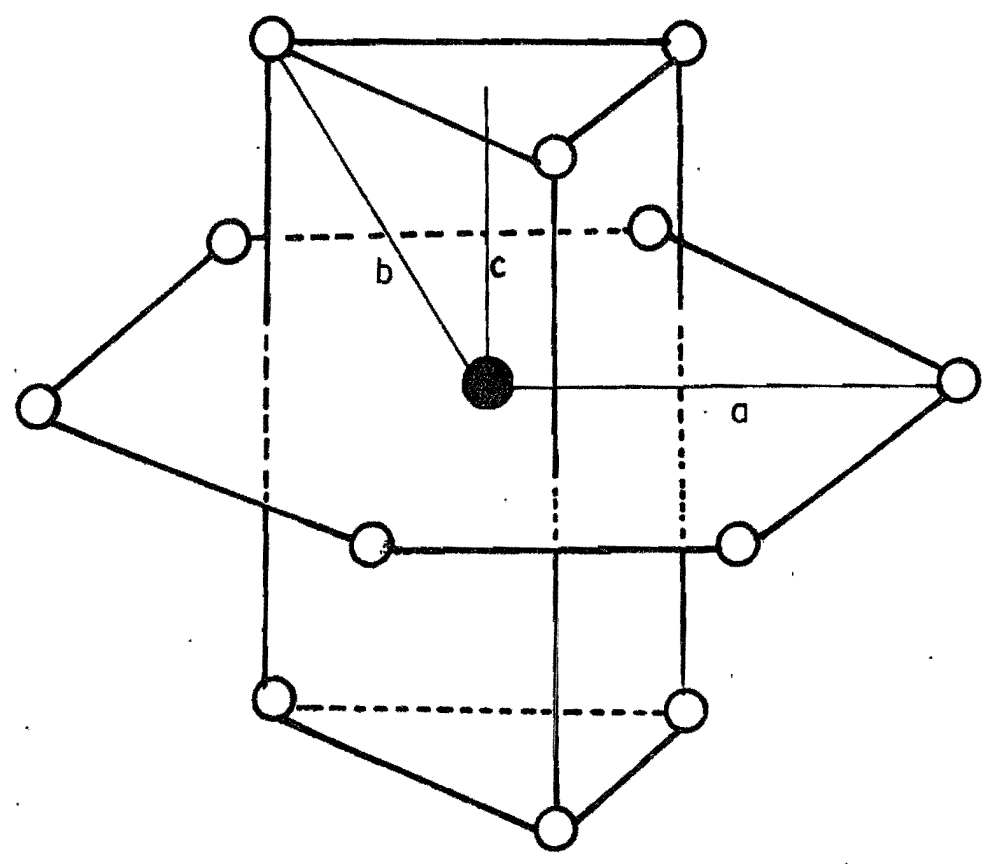

$$
\begin{aligned}
& \eta_{1}=c / 2 b \\
& \eta_{2}=\left[1-\eta_{1}^{2}\right]^{1 / 2}
\end{aligned}
$$

Figure 4. Interatomic distances in an hcp impurity cluster. 


\section{NONDEGENERATE MODES}

In this section we shall simplify Eq.(3.2.4) under the assumption of central forces.

Equations (5.1.20) enable us to write the matrix $\delta l_{A_{2}^{\prime \prime}}$ of $E q \cdot(4.11)$ in the form

$$
\delta l_{A z^{\prime \prime}}=\left[\begin{array}{cccc}
\delta l_{x x}(0,0) & 0 & \sqrt{6} \delta l_{x x}(0,8) & 2 \sqrt{6}\left(\eta_{2} / \eta_{2}\right) \delta l_{x x}(0,8) \\
0 & 0 & 0 & 0 \\
\sqrt{6} \delta l_{x x}(0 ; 0) & 0 & -\delta l_{x x}(0,8) & 2 \sqrt{6}\left(\eta_{2} / \eta_{1}\right) \delta l_{x x}(0,8) \\
2 \sqrt{6} \delta l_{x x}(0,0) & 0 & -2 \delta l_{x x}(0 ; 8) & 4\left(\eta_{2} / \eta_{1}\right) \delta l_{x z}(0,8) \\
\end{array}\right] .
$$

By using the various elements $\left\{\Phi_{\alpha \beta}\left(l_{*}, l^{\prime} \kappa^{\prime}\right)\right\}$ in Eq. (5.1.14) it can be shown that

$$
\Phi_{\alpha \beta}(0,0)=-6\left[\Phi_{\alpha \beta}(0,1)+\Phi_{\alpha \beta}(0,-8)\right] \text {. }
$$

From Eq. (2.2.3) we obtain

$$
\begin{aligned}
\delta l_{x x}(0 ; 0) & =\epsilon M \omega^{2}+\Phi_{x x}(0 ; 0)-\Phi_{x x}^{0}(0 ; 0) \\
& =\epsilon M \omega^{2}+6\left[\Phi_{x x}^{0}(0 ; 8)-\Phi_{x x}(0,8)\right] \\
& =\epsilon M \omega^{2}+6 \lambda(b) \Phi_{x x}^{0}(0 ; 8) \\
& =\epsilon M \omega^{2}-\lambda(b) \Phi_{x x}^{0}(0 ; 0) \\
& =\epsilon M \omega^{2}-\lambda_{2} \Phi_{x x}^{0}(0 ; 0)
\end{aligned}
$$

Here we have used the fact that $\Phi_{x x}(0,1)=0$. Now Eq. (5.2.1) can be rewritten as 


$$
\delta l_{A_{2}{ }^{\prime}}=\left[\begin{array}{cccc}
\epsilon M \omega^{2}-\lambda_{2} \Phi_{x x}^{0}(0,0) & 0 & -\sqrt{6} \lambda_{2} \Phi_{x x}^{0}(0,8) & -2 \sqrt{6} \lambda_{2}\left(\eta_{2} / \eta_{1}\right) \Phi_{x x}^{0}(0,8) \\
0 & 0 & 0 & 0 \\
-\sqrt{6} \lambda_{2} \Phi_{x x}^{0}(0,8) & 0 & \lambda_{2} \Phi_{x x}^{0}(0,8) & 2 \lambda_{2}\left(\eta_{2} / \eta_{1}\right) \Phi_{x x}^{0}(0,-8) \\
-2 \sqrt{6} \lambda_{2} \Phi_{x z}^{0}(0,8) & 0 & 2 \lambda_{2} \Phi_{x z}^{0}(0,8) & 4 \lambda_{2}\left(\eta_{2} / \eta_{1}\right) \Phi_{x z}^{0}(0,8)
\end{array}\right]
$$

Eq. (2.1.20) gives us the following Green's functions' relations:

$$
\begin{aligned}
\Phi_{x x}^{0}(0 ; 0) g_{0} & +6\left[\Phi_{x x}^{0}(0 ; 1) g_{1}+\Phi_{x x}^{0}(0,-8) g_{2}+\Phi_{x x}^{0}(0 ; 8) g_{3}\right] \\
= & {\left[M \omega^{2} g_{0}-1\right], } \\
\Phi_{x x}^{0}(0 ; 0) g_{1} & +\Phi_{x x}^{0}(0 ; 1) A+2 \Phi_{x x}^{0}(0 ; 8) B+\Phi_{x z}^{0}(0 ; 8) C \\
= & M \omega^{2} g_{1}, \\
\Phi_{x x}^{0}(0 ; 0) g_{2} & +2 \Phi_{x x}^{0}(0 ; 1) B+\Phi_{x x}^{0}(0,8) D+2 \Phi_{x z}^{0}(0 ; 8) g_{4} \\
= & M \omega^{2} g_{2}, \\
& +\Phi_{x x}^{0}(0,-1) C+2 \Phi_{x x}^{0}(0,8) g_{4}+\Phi_{x x}^{0}(0 ; 8) F \\
\Phi_{x x}^{0}(0 ; 0) g_{3} & +M \omega^{2} g_{3}
\end{aligned}
$$

The functions $g_{0}, g_{1}, g_{2}, g_{3}, g_{4}, A, B, C, D$, and $F$ are defined in Eqs. (4.13). In the central force calculation $\Phi_{x x}^{0}(0,1)=0$ in all the relations above.

Using $g_{A_{2}^{\prime}}$ given in Eq. (4.10) and $\delta l_{A_{2}^{\prime \prime}}$ given in Eq. $(5.2 .4)$ and substituting from Eqs. (5.2.5) - (5.2.8), we obtain: 
55

$$
g_{A_{2}^{\prime \prime}} \delta l_{A_{2}^{\prime \prime}}=\left[\begin{array}{cccc}
g_{0}\left(\theta-M \omega^{2}\right)+1 & 0 & (1 / \sqrt{6})\left(M \omega g_{0}-1\right) & (2 / \sqrt{6})\left(\eta_{2} / \eta_{1}\right)\left(M \omega^{2} g_{0}-1\right) \\
\sqrt{6} g_{1}\left(\theta-M \omega^{2}\right) & 0 & M \omega^{2} g_{1} & 2\left(\eta_{2} / \eta_{2}\right) M \omega^{2} g_{1} \\
\sqrt{6} g_{2}\left(\theta-M \omega^{2}\right) & 0 & M \omega^{2} g_{2} & 2\left(\eta_{2} / \eta_{1}\right) M \omega^{2} g_{2} \\
\sqrt{6} / 2 g_{3}\left(\theta-M \omega^{2}\right) & 0 & M \omega^{2} g_{3} & \left(\eta_{2} / \eta_{1}\right) M \omega^{2} g_{3}
\end{array}\right],
$$

where $\theta=\in M \omega^{2} / \lambda_{2}$.

The condition that Eq. (3.2.4) has nontrivial solutions is that the secular determinant of the coefficients vanishes:

$$
\Delta_{A_{2}^{\prime \prime}}\left(\omega^{2}\right)=\left|1-g_{A_{2}^{\prime \prime}} \delta l_{A_{2}^{\prime \prime}}\right|=0 \text {. }
$$

After substituting from Eq. $(5.2 .9)$ in Eq. (5.2.11) we obtain

$$
M \omega^{2} g_{0}=\left\{\frac{\lambda_{2}(1-\epsilon) M \omega^{2}}{\Phi_{k x}^{0}(0 ; 0)}+\left(1-\lambda_{2}\right)\right\} /\left\{\frac{\lambda_{2}(1-\epsilon) M \omega^{2}}{\Phi_{x x}^{0}(0 ; 0)}-\epsilon\left(1-\lambda_{2}\right)\right\}
$$

If we define

$$
\rho_{x x}\left(\omega^{2}\right)=\frac{\epsilon}{1-\epsilon}-\frac{M \omega^{2} \lambda_{2}}{\left[\Phi_{x x}^{0}(0 ; 0)\right]\left[1-\lambda_{2}\right]},
$$

and

$$
S_{x x}\left(\omega^{2}\right)=\left[M \omega^{2} g_{0}-1\right],
$$


then Eq. $(5.2 .12)$ can be rewritten as:

$$
\left[1-S_{x x}\left(\omega^{2}\right) S_{x x}\left(\omega^{2}\right)\right]=0
$$

Eq. $(5.2 .15)$ is the condition in Eq. $(5,2.11)$ stated in a suitable notation. It gives a resonance or a localized mode in the direction $x$ (22).

From Eq. (5.2.9) we can obtain the $(1,1)$ element of the matrix $\left(I-g_{A^{\prime}} \delta I_{A^{\prime \prime} 2}\right)^{-1}$. This element will be the element $G_{x x}\left(O ; O ; \omega^{2}\right)$ of the Green's function matrix for the imperfect crystal. The expression for $\mathrm{G}_{\mathrm{xx}}\left(0 ; \mathrm{O} ; \omega^{2}\right)$ which we obtain is

$G_{x x}\left(O ; O ; \omega^{2}\right)=\left\{\frac{1-\left[\left(1-M \omega^{2} g_{0}\right)\left(1-\rho_{x x}\left(\omega^{2}\right)+\epsilon /(1-\epsilon)\right)\right]}{M \omega^{2}(1-\epsilon)\left[1-\rho_{x x}\left(\omega^{2}\right) S_{x x}\left(\omega^{2}\right)\right]}\right\}$

It is interesting to note that Eq. $(5 \cdot 2.15)$ and $(5.2 .16)$ have been obtained by Mannheim and Cohen for cubic systems. The definitions of the functions $S\left(\omega^{2}\right)$ and $\rho\left(\omega^{2}\right)$ in the case of cubic systems do not need the labels of Cartesian coordinates since $x, y$, and $z$ axes are equivalent (13). In the case of an HCP crystal we have to define $S_{x x}\left(\omega^{2}\right)$ and $\rho_{\mathrm{xx}}\left(\omega^{2}\right)$. (22).

If $\Phi_{x x}^{0}(0,0)$ is known, one can obtain $g_{0}$ from Eq. (5.2.12). Then the use of Eq. (5.2.16) in Eq. (2.2.22) will give an expression for $\left|X_{x}^{2}(0 ; 0)\right|$. Substitution of $g_{0}$ and $\left|X_{x}^{2}(0 ; 0)\right|$ in Eq. (2.2.23) will give the mean square displacement $\left\langle u_{x}^{2}(0)\right\rangle$.

Equation $(3.2 .5 \mathrm{~b})$ is eight dimensional. Although a central force calculation in this case can be done, it becomes very difficult. Since very general results for the case of central forces are now avallable (22), we have not simplified Eq. (3.2.5b) further by assuming central forces. 
As remarked earlier, Eq. $(3,2,5 \mathrm{~b})$ which is obtained in an exact form in Chapter IV is expected to be of importance in a numerical calculation taking into account central and non central forces. 


\section{CHAPTER VI}

\section{CONCLUSION AND RECOMMENDATIONS}

By going through the contents of the various chapters outlined in the introduction and after having a brief look at the rest of the material, one will notice that more emphasis has been given on the mathematical aspects of the problem and instead of considering the dynamics of the crystals $w$ ith point defects in general, a detailed discussion on the steps involved in examining the HCP structure is given. The physical aspects have been considered whenever possible. It must be admitted that in spite of many attempts to supplement the mathematics with the physical background, some readers may still find it purely mathematical at a few places.

There are several reasons for completing this report in the present form. Most of the work on this problem involved calculations, the methods for which are well established in principle. In literature detailed calculations are rarely found. The author had many difficulties at different stages of the work. The temptation to explain the calculations consumed so much length that the general discussion on the subject had to be restricted to stating the problem clearly in the second chapter. A large part of the work is based on group theoretical techniques which cannot be explained in this report and someone unfamiliar with them will have to refer to the textbooks cited. It must be noted that the amount of work involved is considerably large, as compared to. what has been" 
presented. The increase in the amount of mathematics adversely affected the discussions.

It is hoped that the results of Chapter IV will be of use in a numerical calculation in which one can take into account very general force constants. The results obtained for the central forces is an illustration of more analytical simplification. The central force calculations were not carried out because during the preparation of this work Cohen (22) reported very general results applicable to various crystal structures under the special assumptions of the nearest neighbour interaction and the central forces.

One possible way to simplify the results of Chapter IV analytically is the use of isotropic changes of force i.e. $\Delta \phi^{\prime \prime}(r)=\frac{\Delta \phi^{\prime}(r)}{r}$ (9).

By following the method used in reference 13 , one can use the results of Chapter IV to obtain the linear response function $K(\omega)$ of the system to an external probe. 


\section{BIBLIOGRAPHY}

A. General References:

The references which were useful in understanding various aspects

of the problem are listed here. They are not cited in the text.

I. E. B. Wilson, Jr., J. C. Decious, and P. C. Cross, Molecular Vibrations, (McGraw-Hill Book Company, New York, 1955), Chapters 5 and 6 .

II. P. M. A. Sherwood, Vibrational Spectroscopy of Solids, (Cambridge University Press, Cambridge, 1972), p. 46.

III. Gerhard Herzberg, Molecular Spectra and Molecular Structure, (D. Van Nostrand Company, Inc., New York, 1945), p. 131.

IV. J. C. Slater, Quantum Theory of Molecules and Solids, Vol.2, (McGraw -Hill Book Company, New York, 1965), p. 32.

V. R. S. Knox and Albert Gold, Symmetry in the Solid State, (W. A. Benjamin, Inc., New York, 1964).

B. References Cited:

1. I. M. Lifshitz, J. Phys. USSR 7, 215,(1943).

2. I. M. Lifshitz, Advan. Phys. 13, 483, (1964).

3. A. A. Maradudin, in Solid State Physics edited by F. Seitz and D. Turnbull (Academic Press, New York, 1966), Vol. 18, p. 273 and Vol. 19, p. 1 .

4. A. A. Maradudin, Rep. Prog. Phys. 28, 331, (1965).

5. R. J. Elliot in Phonons edited by R. W. H. Stevenson, (Plenum Press, New York, 1966), p. 377. .......... Vibrations of Defects in Lattices, ANL-7237, Physics (TID-4500), AEC Research and Development Report, 1966.

6. A. A. Maradudin, E. W. Montroll, G. H. Weiss, and I. P. Ipatowa, Theory of Lattice Dynamics in the Harmonic Approximation, Second Edition (Academic Press, New York, 1971) p. 353.

7. Simon S. Cohen, D. G. Howard, R. H. Nussbaum, To be published in Rep. Prog. Phys. (This review ar ticle will contain an up to date account of the theoretical and experimental developments in the field of the dynamics of a substitutional impurlty in metals. The author is thankful to Professor R. H. Nussbaum 
for giving permission to cite this reference.)

8. P. G. Dawber and R. J. Elliot, Proc. Roy. Soc. (London), 273A, $222,(1963)$.

9. G. W. Lehman and R. E. DeWames, Phys. Rev. 131, 1008, (1963).

10. W. M. Visscher, Phys. Rev. 129, 28, (1963).

11. T. P. Martin, Phys. Rev. 160, 686, (1967).

12. Philip D. Mannheim, Phys. Rev. $\underline{165}$, 1011, (1968).

13. Philip D. Mannheim and Simon S. Cohen, Phys. Rev. B $\underline{4}, 3748$, (1971) .

14. K. Lakatos and J. Krumhansl, Phys. Rev. $175,841,(1968)$.

15. K. Dettman and W. Ludwig, Physik Condensierten Materie $\underline{2}$, $241,(1964)$.

16. A. A. Maradudin, Astrophysics and the Many Body Problem, (W. A. Benjamin, Inc., New York, 1963) p. 109.

17. Reference 6, p. 365 .

18. Max Born and Kun Huang, Dynamical Theory of Crystal Lattices, (Oxford, 1954), p. 42 .

19. Michael Tinkham, Group Theory and Quantum Mechanics, (McGrawHill Book Company, New York, 1964).

20. Lowell H. Hall, Group Theory and Symmetry in Chemistry, (McGraw-Hill Book Company, New York, 1969), p. 136.

21. F. Albert Cotton, Chemical Applications of Group Theory, Second Edition (Wiley - Interscience, New York, 1971), p. 295.

22. Simon S. Cohen, To be published. 


\section{APPENDIX I}

Two dimensional irreducible representations $\Gamma^{E^{\prime}}$ and $\Gamma^{E^{\prime \prime}}$ of the point group $D_{3 h}$ are given below. They can be obtained by considering the transformations of a two dimensional vector $r$ under the various symmetry operations of $D_{3 h}$ and keeping in mind the desired character system. It should be noted that the transposes of the matrices which are listed below can also be used. They are physically equivalent. To avoid confusion one has to use the irreducible representations corsistently.

Representation E':

$$
\begin{aligned}
& \Gamma^{\boldsymbol{\sigma}^{\prime}}(\mathrm{E})=\left[\begin{array}{l}
1 \\
0
\end{array}\right. \\
& \left.\begin{array}{ll}
0 \\
1
\end{array}\right] \\
& \Gamma{ }^{E^{\prime}}\left(\sigma_{h}\right)=\left[\begin{array}{l}
1 \\
0
\end{array}\right. \\
& 1 \\
& \Gamma^{E^{\prime}}\left(C_{3}\right)=\left[\begin{array}{ll}
-1 / 2 & -\sqrt{3} / 2 \\
\sqrt{3} / 2 & -1 / 2
\end{array}\right] \\
& \Gamma^{\Sigma^{\prime}}\left(S_{3}\right)=\left[\begin{array}{ll}
-1 / 2 & -\sqrt{3} / 2 \\
\sqrt{3} / 2 & -1 / 2
\end{array}\right] \\
& \stackrel{\boldsymbol{E}^{\prime}}{\Gamma}\left(\mathrm{C}_{3}^{-1}\right)=\left[\begin{array}{ll}
-1 / 2 & \sqrt{3} / 2 \\
-\sqrt{3} / 2 & -1 / 2
\end{array}\right] \\
& \Gamma^{\boldsymbol{e}^{\prime}}\left(\mathrm{S}_{3}^{-1}\right)=\left[\begin{array}{cc}
-1 / 2 & \sqrt{3} / 2 \\
-\sqrt{3} / 2 & -1 / 2
\end{array}\right] \\
& \left.\Gamma^{E^{\prime}}(1)\right)=\left[\begin{array}{l}
1 \\
0
\end{array}\right. \\
& \left.\begin{array}{ll}
0 \\
1
\end{array}\right] \\
& \Gamma^{E^{\prime}}\left(C_{2}^{\prime \prime}(1)\right)=\left[\begin{array}{l}
1 \\
0
\end{array}\right. \\
& 0 \\
& -1 \\
& \Gamma^{e^{\prime}}\left(\sigma_{v}(2)\right)=\left[\begin{array}{l}
-1 / 2 \\
-\sqrt{3} / 2
\end{array}\right. \\
& -\sqrt{3} / 2] \\
& \Gamma^{E^{\prime}}\left(C_{2}^{\prime \prime}(2)\right)=\left[\begin{array}{l}
-1 / 2 \\
-\sqrt{3} / 2
\end{array}\right. \\
& \left.\begin{array}{l}
-\sqrt{3} / 2 \\
1 / 2
\end{array}\right]
\end{aligned}
$$


Appendix I

Representation E' (continued):

$$
\Gamma^{E^{\prime}}\left(\sigma_{3}(3)\right)=\left[\begin{array}{cc}
-1 / 2 & \sqrt{3} / 2 \\
\sqrt{3} / 2 & 1 / 3
\end{array}\right] \cdot \Gamma_{\left(C_{2}^{\prime \prime}(3)\right)}^{E^{\prime}}=\left[\begin{array}{cc}
-1 / 2 & \sqrt{3} / 2 \\
\sqrt{3} / 2 & 1 / 2
\end{array}\right]
$$

Representation $\mathrm{E}^{\prime \prime}$ :

$$
\begin{aligned}
& \Gamma^{\varepsilon^{u}}(\mathrm{E})=\left[\begin{array}{ll}
1 & 0 \\
0 & 1
\end{array}\right] \quad \Gamma^{\mathrm{E}^{*}}\left(\sigma_{h}\right)=\left[\begin{array}{cc}
-1 & 0 \\
0 & -1
\end{array}\right] \\
& \Gamma^{\varepsilon^{\prime}}\left(C_{3}\right)=\left[\begin{array}{cc}
-1 / 2 & -\sqrt{3} / 2 \\
\sqrt{3} / 2 & -1 / 2
\end{array}\right] \quad \Gamma^{\varepsilon^{\prime \prime}}\left(S_{3}\right)=\left[\begin{array}{cc}
1 / 2 & \sqrt{3} / 2 \\
-\sqrt{3} / 2 & 1 / 2
\end{array}\right] \\
& \Gamma^{E^{\prime \prime}}\left(C_{3}^{-1}\right)=\left[\begin{array}{ll}
-1 / 2 & \sqrt{3} / 2 \\
-\sqrt{3} / 2 & -1 / 2
\end{array}\right] \quad \Gamma^{E^{\prime \prime}}\left(S_{3}^{-1}\right)=\left[\begin{array}{ll}
1 / 2 & -\sqrt{3} / 2 \\
\sqrt{3} / 2 & 1 / 2
\end{array}\right] \\
& \Gamma^{\varepsilon^{\prime \prime}}\left(C_{2}^{\prime \prime}(1)\right)=\left[\begin{array}{cc}
1 & 0 \\
0 & -1
\end{array}\right] \quad \Gamma^{\varepsilon^{\prime \prime}}\left(\sigma_{b}(1)\right)=\left[\begin{array}{ll}
-1 & 0 \\
0 & 1
\end{array}\right] \\
& \Gamma^{E^{\prime \prime}}\left(C_{2}^{\prime \prime}(2)\right)=\left[\begin{array}{ll}
-1 / 2 & -\sqrt{3} / 2 \\
-\sqrt{3} / 2 & 1 / 2
\end{array}\right] \quad \Gamma^{E^{\prime \prime}}\left(\sigma_{v}(2)\right)=\left[\begin{array}{cc}
1 / 2 & \sqrt{3} / 2 \\
\sqrt{3} / 2 & -1 / 2
\end{array}\right] \\
& \Gamma^{E^{*}}\left(C_{2}^{\prime \prime}(3)\right)=\left[\begin{array}{cc}
-1 / 2 & \sqrt{3} / 2 \\
\sqrt{3} / 2 & 1 / 2
\end{array}\right] \quad \Gamma^{z^{\prime \prime}}\left(\sigma_{b}(3)\right)=\left[\begin{array}{cc}
1 / 2 & -\sqrt{3} / 2 \\
-\sqrt{3} / 2 & -1 / 2
\end{array}\right]
\end{aligned}
$$




\section{APPENDIX II}

The matrix operators $S$ referred to in the third section of Chapter II are listed below. These are active operators and the coordinate system chosen is the same as the one used in Chapter III.

$S(E)=\left[\begin{array}{lll}1 & 0 & 0 \\ 0 & 1 & 0 \\ 0 & 0 & 1\end{array}\right] \quad S\left(\sigma_{h}\right)=\left[\begin{array}{ccc}-1 & 0 & 0 \\ 0 & 1 & 0 \\ 0 & 0 & 1\end{array}\right]$

$s\left(C_{3}\right)=\left[\begin{array}{ccc}1 & 0 & 0 \\ 0 & -1 / 2 & -\sqrt{3} / 2 \\ 0 & -\sqrt{3} / 2 & -1 / 2\end{array}\right]$

$S\left(S_{3}\right)=\left[\begin{array}{ccc}-1 & 0 & 0 \\ 0 & -1 / 2 & -\sqrt{3} / 2 \\ 0 & 3 / 2 & -1 / 2\end{array}\right]$

$S\left(C_{3}^{-1}\right)=\left[\begin{array}{ccc}1 & 0 & 0 \\ 0 & -1 / 2 & \sqrt{3} / 2 \\ 0 & -5 / 2 & -1 / 2\end{array}\right]$

$S\left(S_{3}^{-1}\right)=\left[\begin{array}{ccc}-1 & 0 & 0 \\ 0 & -1 / 2 & \sqrt[3]{3} / 2 \\ 0 & -\sqrt{3} / 2 & -1 / 2\end{array}\right]$

$S\left(\sigma_{0}(1)\right)=\left[\begin{array}{ccc}1 & 0 & 0 \\ 0 & -1 & 0 \\ 0 & 0 & 1\end{array}\right]$

$S\left(C_{2}(1)\right)=\left[\begin{array}{rrr}-1 & 0 & 0 \\ 0 & -1 & 0 \\ 0 & 0 & 1\end{array}\right]$

$S\left(\sigma_{0}(2)\right)=\left[\begin{array}{ccc}1 & 0 & 0 \\ 0 & 1 / 2 & \sqrt{3} / 2 \\ 0 & \sqrt{3} / 2 & -1 / 2\end{array}\right]$

$S\left(C_{2}^{\prime \prime}(2)\right)=\left[\begin{array}{ccc}-1 & 0 & 0 \\ 0 & 1 / 2 & \sqrt{3} / 2 \\ 0 & \sqrt{3} / 2 & -1 / 2\end{array}\right]$ 
65

Appendix II (continued)

$$
S\left(\sigma_{v}(3)\right)=\left[\begin{array}{ccc}
1 & 0 & 0 \\
0 & 1 / 2 & -\sqrt{3} / 2 \\
0 & -\sqrt{3} / 2 & -1 / 2
\end{array}\right] \quad S\left(C_{2}^{\prime}(3)\right)=\left[\begin{array}{ccc}
-1 & 0 & 0 \\
0 & 1 / 2 & -\sqrt{3} / 2 \\
0 & -\sqrt{3} / 2 & -1 / 2
\end{array}\right]
$$




\section{APPENDIX III}

In this appendix we illustrate the use of Eq. (2.3.16), which is reproduced below, to determine the linearly independent elements of the matrix G:

$$
G^{0}\left(L_{1} K_{1} ; L_{2} K_{2} ; \omega^{2}\right)=S(R) G^{0}\left(1_{1} k_{1} ; 1_{2} k_{2} ; \omega^{2}\right) S^{T}(R), \quad(A 3.1)
$$

where $S^{T}(R)$ is the transpose of the matrix representation $S(R)$. These matrices are listed in Appendix II. The space group operation R takes atoms at the sites $\left(l_{1} \kappa_{1}\right)$ and $\left.I_{2} K_{2}\right)$ to the sites $\left(L_{1} K_{1}\right)$ and $\left(L_{2} K_{2}\right)$ respectively. For determining the independent elements of $G^{0}\left(1_{1} k_{1} ; 1_{2} k_{2}, \omega^{2}\right)$ the best way is to choose the operation that leaves both the sites $\left(1_{1} k_{1}\right)$ and $\left(1_{2} k_{2}\right)$ unshifted. The symmetry operation $\sigma_{h}$ leaves sites $0,1,2, \ldots 6$ unshifted. Hence for $\mathrm{G}^{0}(0,1)$ we can write:

$$
G^{0}\left(0,1, \omega^{2}\right)=S\left(\sigma_{h}\right) G^{0}\left(0,1, \omega^{2}\right) S^{T}\left(\sigma_{h}\right) \text {. }
$$

After solving the right hand side of (A3.2) it can be seen that the $x y, x z$, $y x$, and $z x$ elements change sign. Since the two sides must be identical these elements must be zero. Hence $G(0,1)$ has the form

$$
\left[\begin{array}{ccc}
G_{x x}^{0}(0 ; 1) & 0 & 0 \\
0 & G_{y y}^{0}(0 ; 1) & G_{z z}^{0}(0 ; 1) \\
0 & G_{z y}^{0}(0 ; 1) & G_{z z}^{0}(0 ; 1)
\end{array}\right]
$$

Other matrices $G^{0}\left(n_{1} ; n_{2}\right)$ where $n_{1} ; n_{2}=0,1,2, \ldots 6$ will have the same form. 
The sites $l_{k}=2,3, \ldots 6$ can be reached from the site $I_{k}=1$ by waing various operations of $D_{3 h}$. Hence all $G^{0}(0, n)$ matrices for $n=2$, $3, \ldots 6$ can be expressed in terms of $G^{0}(0,1)$. For example:

$$
\begin{aligned}
& \dot{G}^{0}(0,2)=S\left(\sigma_{1}(3)\right) G^{0}(0,1) S^{\top}\left(\sigma_{0}(3)\right) \\
& =\left[\begin{array}{ccc}
G_{x x}^{0}(0,1) & 0 & 0 \\
0 & \frac{1}{4}\left[\left(G_{y y}^{0}+3 G_{z z}^{0}\right)\right. & -\frac{1}{4}\left[\sqrt{3}\left(G_{y y}^{0}-G_{z z}^{0}\right)\right. \\
& \left.-\sqrt{3}\left(G_{y z}^{0}+G_{z y}^{0}\right)\right] & \left.+\left(G_{y z}^{0}-3 G_{z y}^{0}\right)\right] \\
& -\frac{1}{4}\left[\sqrt{3}\left(G_{y y}^{0}-G_{z z}^{0}\right)\right. & +\frac{1}{4}\left[\left(3 G_{y y}^{0}+G_{z z}^{0}\right)\right. \\
& \left.-\left(3 G_{y z}^{0}-G_{z y}^{0}\right)\right] & \left.+\sqrt{3}\left(G_{y z}^{0}+G_{z y}^{0}\right)\right]
\end{array}\right]
\end{aligned}
$$

where all the $G^{\prime} s$ in the matrix on the right hand side are $G_{\alpha \beta}^{0}\left(0^{\circ} ; 1\right)$. The site indices are dropped to save the space.

Since $\mathrm{g}$ is a submatrix of $\mathrm{G}^{0}$, all the elements of $\mathrm{g}$ can be expressed as linear combinations of its linearly independent elements by using the method used above. One must remember that while dealing with the $\mathrm{g}$ matrix one can use the space group operations.

For determination of the linearly independent elements of $\delta 1$, one follows the same procedure only with the difference that since $\delta 1$ refers to the perturbed crystal, one has to use the point group operations. The perturbed crystal does not have the translational symmetry. 


\section{APPENDIX IV}

\section{DETERMINATION OF SALC'S}

For analyzing the normal modes of vibration of the impurity cluster considered in Chapter III, we need the basis vectors for each of the irreducible representation appearing in Eq. (3.1.3). In Chapter IV we considered $4 \dot{A}_{2}^{\prime \prime}$ and $8 E^{\prime}$ modes in which the defect moves. In this appendix, we obtain the basis vectors for all the irreducible representations in Eq. (3.1.3). These basis vectors are referred to as the symmetry adapted linear combinations (SALC's) .(21).

We define the projection operator for the $j^{\text {th }}$ irreducible representation as:

$$
\rho_{\mu \mu}^{(j)}=\left(1_{j} / h\right) \sum_{R} \Gamma_{\mu \mu}^{(j)}(R) P_{R}
$$

where $l_{j}$ is the dimensionality of the $j^{\text {th }}$ irreducible representation, $h$ is the total number of the elements in the point group, $\Gamma^{(j)}(R)$ is the $j^{\text {th }}$ irreducible representation of the symmetry operation $R$, and $P_{R}$ is the active operator which performs the symmetry operation on the crystal. This projection operator based on the $\mu^{\text {th }}$ row of the $j^{\text {th }}$ irreducible representation projects from an arbitrary function $\psi$ another function $\psi_{\mu}^{(j)}$ such that $\psi_{\mu}^{(j)}$ is a linear combination of the basis vectors of the $j^{\text {th }}$ irreducible representation. In other words $\rho_{\mu \mu}^{(j)}$ projects that part of $\psi$ which belongs to the $\mu^{\text {th }}$ row of the $j^{\text {th }}$ irreducible representation. We shall use the thirty-nine displacement coordinates $\left\{u_{\alpha}(n)\right\}$ of the 
impurity cluster as arbitrary functions to be operated by $\rho_{\mu}^{(j)}$. From the results of the operation of $\mathcal{S}_{\mu \mu}^{(j)}$ on $\left\{u_{\alpha}(n)\right\}$ we can select a basis for the $j^{\text {th }}$ irreducible representation. This procedure will also determine whether a particular displacement belongs to the irreducible representation or not. For example:

$$
\int_{\mu \mu}^{(j)} u_{\alpha}(n)=0
$$

implies that the motion of the $n^{\text {th }}$ atom in the direction does not belong to the $j^{\text {th }}$ irreducible representation. Table VI lists the displacements belonging to the various normal modes of the impurity cluster. The basis vectors for various representations are listed in Table VII. 
TABLE VI

DISPLACEMENTS BELONGING TO THE VARIOUS NORMAL MODES OF THE IMPURITY CLUSTER

\begin{tabular}{|c|c|}
\hline Normal Modes & Displacements \\
\hline $4 A_{1}^{\prime}$ & $\begin{array}{l}u_{y}(n) ; u_{z}(n), n=1 \text { to } 6 \\
u_{y}(n) \quad n=7,9,10, \text { or } 12 \\
u_{\alpha}(n) \quad \alpha=x \text { or } z \text { and } n=7 \text { to } 12\end{array}$ \\
\hline $3 A_{2}^{\prime}$ & $\begin{array}{l}u_{y}(n) \quad n=1 \text { to } 12 \\
u_{z}(n) \quad n=1 \text { to } 6 \text { and } 7,9,10 \text { or } 12\end{array}$ \\
\hline $2 \mathrm{~A}_{1}^{\prime}$ & $\begin{array}{ll}u_{x}(n) & n=1 \text { to } 6 \\
u_{y}(n) & n=7 \text { to } 12 \\
u_{z}(n) & n=7,9,10 \text { or } 12\end{array}$ \\
\hline $4 A_{2}^{\prime \prime}$ & $\begin{array}{ll}u_{x}(n) & n=0 \text { to } 12 \\
u_{y}(n) & n=7,9,10 \text { or } 12 \\
u_{z}(n) & n=7 \text { to } 12\end{array}$ \\
\hline $\begin{array}{l}8 E^{\prime} \\
\text { (Modes belonging } \\
\text { to the first row of } E^{\prime} \text { ) }\end{array}$ & $\begin{array}{ll}u_{x}(n) & n=7 \text { to } 12 \\
u_{y}(n) & n=1 \text { to } 12 \\
u_{z}(n) & n=0 \text { to } 7,9,10 \text { or } 12\end{array}$ \\
\hline $\begin{array}{l}8 E^{\prime} \\
\text { (Modes bel onging } \\
\text { to the second row } \\
\text { of } E^{\prime} \text { ) }\end{array}$ & $\begin{array}{l}u_{x}(n) \quad n=7,9,10 \text { or } 12 \\
u_{y}(n) \quad n=1 \text { to } 7,9,10 \text { or } 12 \\
u_{z}(n) \quad n=1 \text { to } 12\end{array}$ \\
\hline
\end{tabular}


TABLE VI (continued)

\begin{tabular}{|lll|}
\hline $5 E^{\prime \prime}$ & $u_{x}(n)$ & $n=1$ to $7,9,10$ or 12 \\
$\begin{array}{ll}\text { (Modes belong- } \\
\text { ing to the first } \\
\text { row of E") }\end{array}$ & $u_{y}(n)$ & $n=7$ to 12 \\
& $u_{z}(n)$ & $n=7,9,10$ or 12 \\
\hline $5 E^{\prime \prime}$ & $u_{x}(n)$ & $n=1$ to 12 \\
$\begin{array}{l}\text { (Modes belong- } \\
\text { ing to the second } \\
\left.\text { row of } E^{\prime \prime}\right)\end{array}$ & $u_{y}(n)$ & $n=7,9,10$, or 12 \\
& $u_{z}(n)$ & $n=7$ to 12 \\
\hline
\end{tabular}


TABLE VII

BASES FOR THE IRREDUCIBLE REPRESENTATIONS

OF POINT GROUP. $D_{3 h}$

Representation

Basis Vectors

$$
\begin{aligned}
& A_{1}^{\prime} \quad \alpha_{1}=(1 / \sqrt{24})\left\{\left(u_{y}(1)-u_{y}(6)\right)-\left(u_{y}(2)-u_{y}(5)\right)\right. \\
& -2\left(u_{y}(3)-u_{y}(4)\right)+\sqrt{3}\left[\left(u_{z}(1)+u_{z}(6)\right)\right. \\
& \left.\left.-\left(u_{z}(2)+u_{z}(5)\right)\right]\right\} \\
& \alpha_{2}=(1 / \sqrt{24})\left\{\sqrt{3}\left[\left(u_{y}(1)-u_{y}(6)\right)+\left(u_{y}(2)-u_{y}(5)\right)\right]\right. \\
& -\left(u_{z}(1)+u_{z}(6)\right)-\left(u_{z}(2)+u_{z}(5)\right)^{-} \\
& \left.+2\left(u_{z}(3)+u_{z}(4)\right)\right\} \\
& \alpha_{3}=(1 / \sqrt{6})\left\{u_{x}(7)-u_{x}(10)+u_{x}(8)-u_{x}(11)+u_{x}(9)-u_{x}(12)\right\} \\
& \alpha_{4}=(1 / \sqrt{24})\left\{\sqrt{3}\left[u_{y}(7)+u_{y}(11)\right)-\left(u_{y}(9)+u_{y}(12)\right)\right] \\
& -\left[\left(u_{z}(7)+u_{z}(10)\right)-2\left(u_{z}(8)+u_{z}(11)\right)\right. \\
& \left.\left.+\left(u_{z}(9)+u_{z}(12)\right)\right]\right\} \\
& A_{1}^{\prime \prime} \quad \alpha_{1}=(1 / \sqrt{6})\left\{\left(u_{x}(1)-u_{x}(6)\right)-\left(u_{x}(2)-u_{x}(5)\right)\right. \\
& \left.+\left(u_{x}(3)-u_{x}(4)\right)\right\} \\
& \alpha_{z}=(1 / \sqrt{24})\left\{u_{y}(7)-u_{y}(10)-2\left(u_{y}(8)-u_{y}(11)\right)\right. \\
& +\left(u_{y}(9)-u_{y}(12)\right)+\sqrt{3}\left[\left(u_{z}(7)-u_{z}(10)\right)\right. \\
& \left.-\left(u_{z}(9)-u_{z}(12)\right]\right\}
\end{aligned}
$$


TABLE VII (continued)

$$
\begin{aligned}
A_{2}^{\prime} \quad \alpha_{1}= & (1 / \sqrt{24})\left\{\left(u_{y}(1)-u_{y}(6)\right)+\left(u_{y}(2)-u_{y}(5)\right)\right. \\
& -2\left(u_{y}(3)-u_{y}(4)\right)+\sqrt{3}\left[\left(u_{z}(1)+u_{z}(6)\right)\right. \\
& \left.\left.+\left(u_{z}(2)-u_{z}(5)\right)\right]\right\} \\
\alpha_{2}= & (1 / \sqrt{24})\left\{\sqrt{3}\left[\left(u_{y}(1)+u_{y}(6)\right)-\left(u_{y}(2)+u_{y}(5)\right)\right]\right. \\
& \left.\left.-\left(u_{z}(1)-u_{z}(6)\right)+\left(u_{z}(2)-u_{z}(5)\right)+2\left(u_{z}(3)-u_{z}(4)\right)\right\}\right\} \\
\alpha_{3}= & (1 / \sqrt{24})\left\{\left(u_{y}(7)+u_{y}(10)\right)-2\left(u_{y}(8)+u_{y}(11)\right)\right. \\
& +\left(u_{y}(9)+u_{y}(12)\right): \\
& \left.+\sqrt{3}\left[u_{z}(7)+u_{z}(10)-\left(u_{z}(9)+u_{z}(12)\right)\right]\right\}
\end{aligned}
$$

$A^{\prime \prime}{ }_{2} \cdot \alpha_{1}=u_{x}(0)$

$$
\begin{aligned}
\alpha_{2}= & (1 / \sqrt{6}) \sum_{n=1}^{6} u_{x}(n) \\
\alpha_{3}= & (1 / \sqrt{6}) \sum_{n=7}^{12} u_{x}(n) \\
\alpha_{4}= & (1 / \sqrt{24})\left\{\sqrt{3}\left[\left(u_{y}(7)-u_{y}(10)\right)-\left(u_{y}(9)-u_{y}(12)\right)\right]\right. \\
& -\left[\left(u_{z}(7)-u_{z}(10)\right)-2\left(u_{z}(8)-u_{z}(11)\right)\right. \\
& \left.\left.+\left(u_{z}(9)-u_{z}(12)\right)\right]\right\}
\end{aligned}
$$

The first

row of $\alpha_{1}=u_{z}(0)$

$$
\begin{aligned}
& \alpha_{2}=(1 / \sqrt{6}) \sum_{n=1}^{6} u_{z}(n) \\
& \alpha_{3}=(1 / \sqrt{6})\left[\left(u_{y}(1)-u_{y}(6)\right)-\left(u_{y}(2)-u_{y}(5)\right)\right. \\
& \left.\quad+\left(u_{y}(3)-u_{y}(4)\right)\right] . \quad \text { (To be contin }
\end{aligned}
$$


Table VII (continued)

\section{The first row of}

$\mathrm{E}^{\prime}$ (continued)

$$
\begin{aligned}
\alpha_{4}= & (1 / \sqrt{10})\left\{\sqrt { 3 } \left(u_{y}(1)-u_{y}(6)+\left[\left(u_{z}(2)+u_{z}(5)\right)\right.\right.\right. \\
& \left.\left.-\left(u_{z}(3)+u_{z}(4)\right)\right]\right\} \\
\alpha_{5}= & (1 / \sqrt{10})\left\{\sqrt{3}\left(u_{z}(1)+u_{z}(6)\right)+\left[u_{y}(2)-u_{y}(5)\right.\right. \\
& \left.\left.+u_{y}(3)-u_{y}(4)\right]\right\} \\
\alpha_{6}= & (1 / \sqrt{6}) \sum_{n=7}^{12} u_{z}(n) \\
\alpha_{7}= & (1 / \sqrt{10})\left\{\sqrt{3}\left(u_{z}(8)+u_{z}(11)\right)\right. \\
& \left.-\left[\left(u_{y}(7)+u_{y}(10)\right)-\left(u_{y}(9)+u_{y}(12)\right)\right]\right\} \\
\alpha_{8}= & 1 / \sqrt{12}\left\{\left(u_{x}(7)-u_{x}(10)\right)-2\left(u_{x}(8)-u_{x}(11)\right)\right. \\
& \left.+\left(u_{x}(9)-u_{x}(12)\right)\right\}
\end{aligned}
$$

The second row of $\mathrm{E}^{\prime}$

$$
\begin{aligned}
\beta_{1}= & u_{y}(0) \\
\beta_{2}= & (1 / \sqrt{6}) \sum_{n=1}^{6} u_{y}(n) \\
\beta_{3}= & (1 / \sqrt{6})\left[\left(u_{z}(1)-u_{z}(6)\right)-\left(u_{z}(2)-u_{z}(5)\right)\right. \\
& \left.+\left(u_{z}(3)-u_{z}(4) \cdot\right)\right] \\
\beta_{4}= & (1 / \sqrt{10})\left\{\sqrt{3}\left(u_{z}(1)-u_{z}(6)\right)-\left[\left(u_{y}(2)+u_{y}(5)\right)\right.\right. \\
& \left.\left.-\left(u_{y}(3)+u_{y}(4)\right)\right]\right\} \\
\beta_{5}= & (1 / \sqrt{10})\left\{\sqrt{3}\left(u_{y}(1)+u_{y}(6)\right)-\left[\left(u_{z}(2)-u_{z}(5)\right)\right.\right. \\
& \left.\left.+\left(u_{z}(3)-u_{z}(4)\right)\right]\right\} \\
= & (1 / \sqrt{6}) \sum_{n=7}^{12} u_{y}(n)
\end{aligned}
$$


Table VII (continued)

The second row

of $E^{\prime}$ (continued)

$$
\begin{aligned}
\beta_{7}= & (1 / \sqrt{10})\left\{\sqrt{3}\left(u_{y}(8)+u_{y}(11)\right)\right. \\
& +\left[\left(u_{z}(7)+u_{z}(10)\right)-\left(\left(u_{z}(9)+u_{z}(12)\right)\right]\right\} \\
= & (1 / 2)\left\{\left(u_{x}(7)-u_{x}(10)\right)-\left(u_{x}(9)-u_{x}(12)\right)\right\}
\end{aligned}
$$

The first row of $E^{\prime \prime}$

$$
\begin{aligned}
\alpha_{1}= & (1 / 2)\left[\left(u_{x}(1)-u_{x}(6)\right)+\left(u_{x}(2)-u_{x}(5)\right)\right] \\
\alpha_{2}= & (1 / 2)\left[\left(u_{x}(1)-u_{x}(6)\right)-\left(u_{x}(3)-u_{x}(4)\right)\right] \\
\alpha_{3}= & (1 / 2)\left[\left(u_{x}(7)+u_{x}(10)\right)-\left(u_{x}(9)+u_{x}(12)\right)\right] \\
\alpha_{4}= & (1 / \sqrt{6})\left[\left(u_{y}(7)-u_{y}(10)+\left(u_{y}(8)-u_{y}(11)\right)\right.\right. \\
& \left.+\left(u_{y}(9)-u_{y}(12)\right)\right] \\
\alpha_{5}= & (1 / \sqrt{10})\left[\sqrt{3}\left(u_{y}(8)-u_{y}(11)\right)+\left(u_{z}(7)-u_{z}(10)\right)\right. \\
& \left.-\left(u_{z}(9)-u_{z}(12)\right)\right]
\end{aligned}
$$

The second row of E"

$$
\begin{aligned}
\alpha_{1}= & (1 / 2)\left[\left(u_{x}(1)+u_{x}(6)\right)-\left(u_{x}(2)+u_{x}(5)\right)\right] \\
\alpha_{2}= & (1 / 2)\left[\left(u_{x}(1)+u_{x}(6)\right)-\left(u_{x}(3)+u_{x}(4)\right)\right] \\
\alpha_{3}= & (1 / \sqrt{12})\left[\left(u_{x}(7)+u_{x}(10)\right)-2\left(u_{x}(8)+u_{x}(11)\right)\right. \\
& \left.+\left(u_{x}(9)+u_{x}(12)\right)\right] \\
\alpha_{4}= & (1 / \sqrt{10})\left[\left(u_{y}(7)-u_{y}(10)\right)-\left(u_{y}(9)-u_{y}(12)\right)\right. \\
& \left.-\sqrt{3}\left(u_{z}(8)-u_{z}(11)\right)\right] \\
\alpha_{5}= & (1 / \sqrt{6})\left[\left(u_{z}(7)-u_{z}(10)\right)+\left(u_{z}(8)-u_{z}(11)\right)\right. \\
& \left.+\left(u_{z}(9)-u_{z}(12)\right)\right]
\end{aligned}
$$




\section{APPENDIX V}

From Eq. (2.1.20) we have obtained eight relations between the elements of the pure lattice Green's function matrix. They can be of use in carrying out analytical calculations for the $8 \mathrm{E}^{\prime}$ modes. We list these relations in this appendix. For simplicity we have used the notation $g_{\alpha \beta}^{n_{1} n_{2}}$ instead of $g_{\alpha \beta}\left(n_{1}, n_{2} \omega^{2}\right)$.

$$
\begin{aligned}
& \left\{g_{y y}^{00} \dot{\Phi}_{y y}^{00}+3\left[g_{y y}^{01} \dot{\Phi}_{y y}^{01}+g_{z z}^{01} \dot{\Phi}_{y y}^{01}+\left(\dot{g}_{y z}^{01}+g_{z y}^{01}\right) \dot{\Phi}_{y z}^{01}+\dot{g}_{y y}^{08} \dot{\Phi}_{y y}^{08}+g_{z z}^{08} \Phi_{z z}^{08}\right.\right. \\
& \left.+g_{x x}^{08} \dot{\Phi}_{x z}^{08}\right\}:=\left[M \omega^{2} \cdot g_{y y}^{00}-1\right] \text {, } \\
& \left\{g_{y y}^{01} \Phi_{y y}^{00}+\left[g_{y y}^{00}-g_{y y}^{01}+g_{y y}^{13}+g_{y y}^{14}-\frac{1}{2} g_{y y}^{15}+\frac{3}{2} g_{z z}^{01}\right] \Phi_{y y}^{01}+\frac{3}{2}\left[g_{y y}^{01}+g_{y y}^{15}\right] \Phi_{z z}^{01}\right. \\
& +\left[g_{y z}^{14}-\frac{1}{2} g_{y z}^{01}+\frac{1}{2} g_{z y}^{01}\right] \Phi_{y z}^{01}+\left[\frac{1}{2} g_{y y}^{08}+\frac{5}{4} g_{y y}^{19}-\frac{3}{4} g_{z z}^{19}+2 g_{y y}^{18}\right] \Phi_{y y}^{08} \\
& \left.+\Phi_{z=}^{08}\left[\frac{3}{2} g_{y y}^{08}+\frac{3}{4} g_{y y}^{19}+\frac{3}{4} g_{z z}^{19}\right]+3 g_{z x}^{19} \Phi_{z x}^{08}\right\}=M \omega^{2} g_{y y}^{01} \text {, } \\
& \left\{g_{z z}^{01} \dot{\Phi}_{y y}^{00}+\frac{3}{2}\left[\dot{g}_{z z}^{01}+g_{z z}^{14}\right] \dot{\Phi}_{y y}^{01}+\left[\frac{1}{2} \dot{g}_{z z}^{11}-\frac{1}{2} g_{z z}^{14}+g_{z z}^{13}+g_{y y}^{00}\right] \dot{\Phi}_{z z}^{01}\right. \\
& +\frac{1}{2}\left(\dot{g}_{y z}^{01}-g_{z y}^{01}\right) \Phi_{z y}^{01}+\left[-g_{z x}^{08}+g_{z x}^{18}-g_{z x}^{19}\right] \Phi_{z x}^{08} \text {. } \\
& \left.+\left[\frac{3}{2} g_{z z}^{08}+\frac{3}{4} g_{y y}^{19}+\frac{3}{4} g_{z z}^{19}\right] \Phi_{y y}^{08}+\left[\frac{1}{2} g_{z z}^{08}+g_{z z}^{18}+\frac{5}{4} g_{z z}^{19}-\frac{3}{4} g_{y y}^{19}\right] \Phi_{z z}^{08}\right\} \\
& =M \omega^{2} G_{z 2}^{01}
\end{aligned}
$$


77

$$
\begin{aligned}
& \left\{\left[g_{y z}^{01} \phi_{y y}^{00}\right]+\left[\frac{1}{2} g_{z y}^{01}+g_{z y}^{14}-\frac{\sqrt{3}}{4}\left(g_{y y}^{15}-g_{z z}^{15}\right)\right] \dot{\Phi}_{y y}^{01}+\frac{3}{2}\left[-g_{y z}^{01}+\frac{\sqrt{3}}{2}\left(g_{y y}^{15}-g_{z z}^{15}\right)\right]\right. \\
& \times \Phi_{z z}^{01}+\left[g_{y y}^{00}-\frac{3}{2} g_{y y}^{01}+\frac{1}{2} g_{z z}^{01}-g_{z z}^{13}+g_{z z}^{14}\right] \Phi_{y z}^{01}+\sqrt{3}\left[g_{z x}^{08}-g_{z x}^{19}\right] \oint_{z x}^{08} \\
& +\left[\frac{\sqrt{3}}{2} \stackrel{08}{g z}_{z z}-\frac{\sqrt{3}}{2} g_{z z}^{19}+2 g_{z y}^{18}+\frac{1}{2} g_{z y}^{19}\right] \Phi_{y y}^{08} \\
& \left.+\left[-\frac{\sqrt{3}}{2}\left(\dot{a}_{z z}^{8}-g_{z z}^{19}\right)+\frac{1}{2} g_{z y}^{19}\right] \Phi_{z z}^{08}\right\} \\
& =M \omega^{2} g_{y_{z}}^{01} \text {, } \\
& \left\{g_{z y}^{01} \Phi_{y y}^{00}+\frac{3}{2}\left[-\frac{\sqrt{3}}{2}\left(g_{y y}^{15}-g_{z z}^{15}\right)-g_{z y}^{01}\right] \Phi_{y y}^{01}+\frac{1}{2}\left[-g_{y z}^{01}+2 g_{z y}^{01}+2 g_{y z}^{14}\right.\right. \\
& \left.+\frac{\sqrt{3}}{2}\left(g_{y y}^{105}-g_{z z}^{105}\right)\right] \Phi_{z z}^{01}+\left[4 g_{y x}^{18}+\sqrt{3} g_{z x}^{19}\right] \phi_{z x}^{08} \\
& +\frac{\sqrt{3}}{2}\left[g_{y y}^{08}-\frac{5}{2} g_{y y}^{19}+\frac{3}{2} g_{z z}^{19}\right] \Phi_{y y}^{08}+\left[-\frac{\sqrt{3}}{2} g_{y y}^{08}+\frac{\sqrt{3}}{4} g_{y y}^{19}+\frac{\sqrt{3}}{4} g_{z z}^{19}\right. \\
& \left.\left.+2 g_{y z}^{18}\right] \Phi_{z z}^{08}\right\}=M \omega^{2} g_{z y}^{01},
\end{aligned}
$$

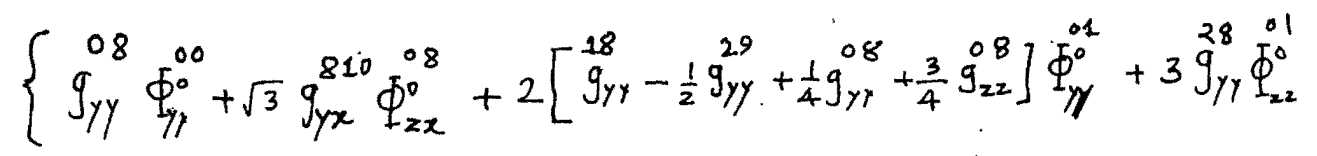

$$
\begin{aligned}
& +\left[\frac{1}{2}\left(g_{y y}^{87}+g_{y y}^{810}\right)+\frac{\sqrt{3}}{2}\left(g_{y z}^{87}+g_{y z}^{810}\right)+\left(g_{y y}^{88}+g_{y y}^{811}\right)\right] \Phi_{y y}^{0.8} \\
& +\left[\frac{3}{2}\left(g_{y y}^{87}+g_{y y}^{810}\right)-\frac{\sqrt{3}}{2}\left(g_{y z}^{87}+g_{y z}^{810}\right)\right] \Phi_{z z}^{08} . \\
& \left.+2\left[g_{y z}^{18}+\frac{\sqrt{3}}{4} g_{y y}^{08}-\frac{\sqrt{3}}{4} g_{z z}^{08}\right] \Phi_{y z}^{01}\right\}=M \omega^{2} g_{y y}^{08},
\end{aligned}
$$


78

$$
\begin{aligned}
& \left\{\left(g_{z x}^{810}+g_{z x}^{88}-g_{z x}^{811}\right) \Phi_{z x}^{08}+2\left(g_{y z}^{18}+\frac{\sqrt{3}}{4} g_{y y}^{08}-\frac{\sqrt{3}}{4} g_{z z}^{08}\right) \Phi_{y z}^{01}+\left[\frac{\sqrt{3}}{2}\left(g_{z y}^{87}+g_{z y}^{810}\right)\right.\right. \\
& \left.+\frac{3}{2}\left(g_{z z}^{87}+g_{z z}^{810}\right)\right] \Phi_{y y}^{08}+\left[-\frac{\sqrt{3}}{2}\left(g_{z y}^{87}+g_{z y}^{810}\right)+\frac{1}{2}\left(g_{z z}^{87}+g_{z z}^{810}\right)\right. \\
& \left.+\left(g_{z z}^{88}+g_{z z}^{811}\right)\right] \Phi_{z z}^{08}+\Phi_{y y}^{00} g_{z z}^{08}+3 g_{z z}^{28} \Phi_{y y}^{01}+2\left[g_{z z}^{18}+\frac{3}{4} g_{y y}^{08}\right. \\
& \left.\left.+\frac{1}{4} g_{z z}^{08}-\frac{1}{2} g_{z z}^{28}\right] \Phi_{z z}^{01}\right\}=M \omega^{2} G_{z z}^{08}, \\
& 08 \\
& g_{x z}^{0} \Phi_{x x}^{00}+\left[2 g_{x z}^{18}+2 g_{x z}^{28}-g_{x z}^{08}\right] \Phi_{x x}^{01}+2\left[g_{z x}^{87}+g_{z x}^{810}\right] \Phi_{x x}^{80} \\
& +\left[\sqrt{3} g_{z y}^{87}-\sqrt{3} g_{z y}^{810}+g_{z z}^{87}-g_{z z}^{810}+g_{z z}^{88}-g_{z z}^{811}\right] \Phi_{x z}^{08} \\
& =M \omega^{2} g_{x z}^{08} .
\end{aligned}
$$




\section{APPENDIX VI}

HCP CRYSTALS

Elements which have hcp structure are listed below with the lattice constants $\mathrm{a}$ and $\mathrm{c}$. Figure 5 indicates their position in the periodic table of elements. (Ref. J. C. Slater, Quantum Theory of Molecules and Solids, Volume 2 (McGraw Hill Book Company, New York, 1965), p. 340 .

TABLE VIII

Hcp Structures

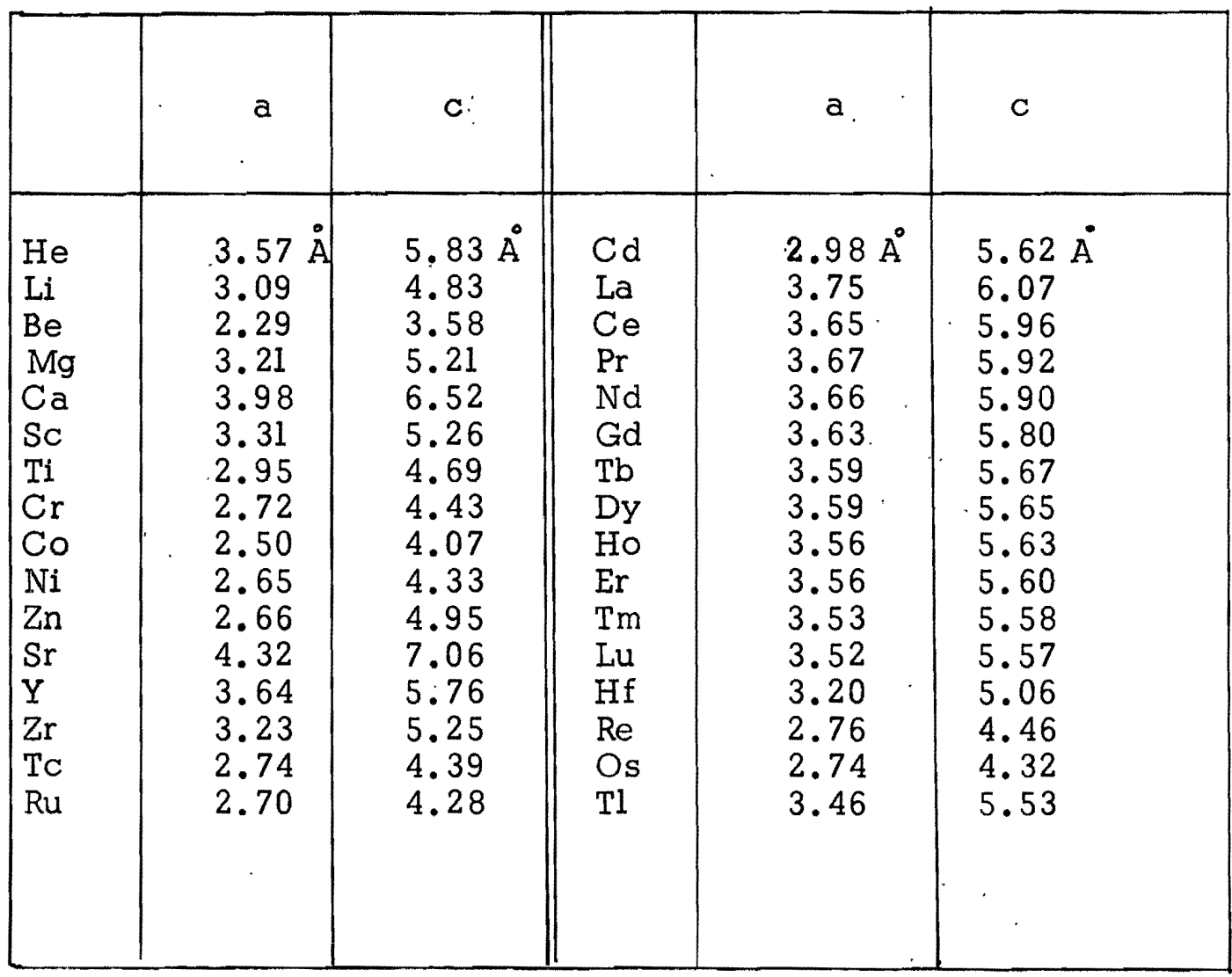




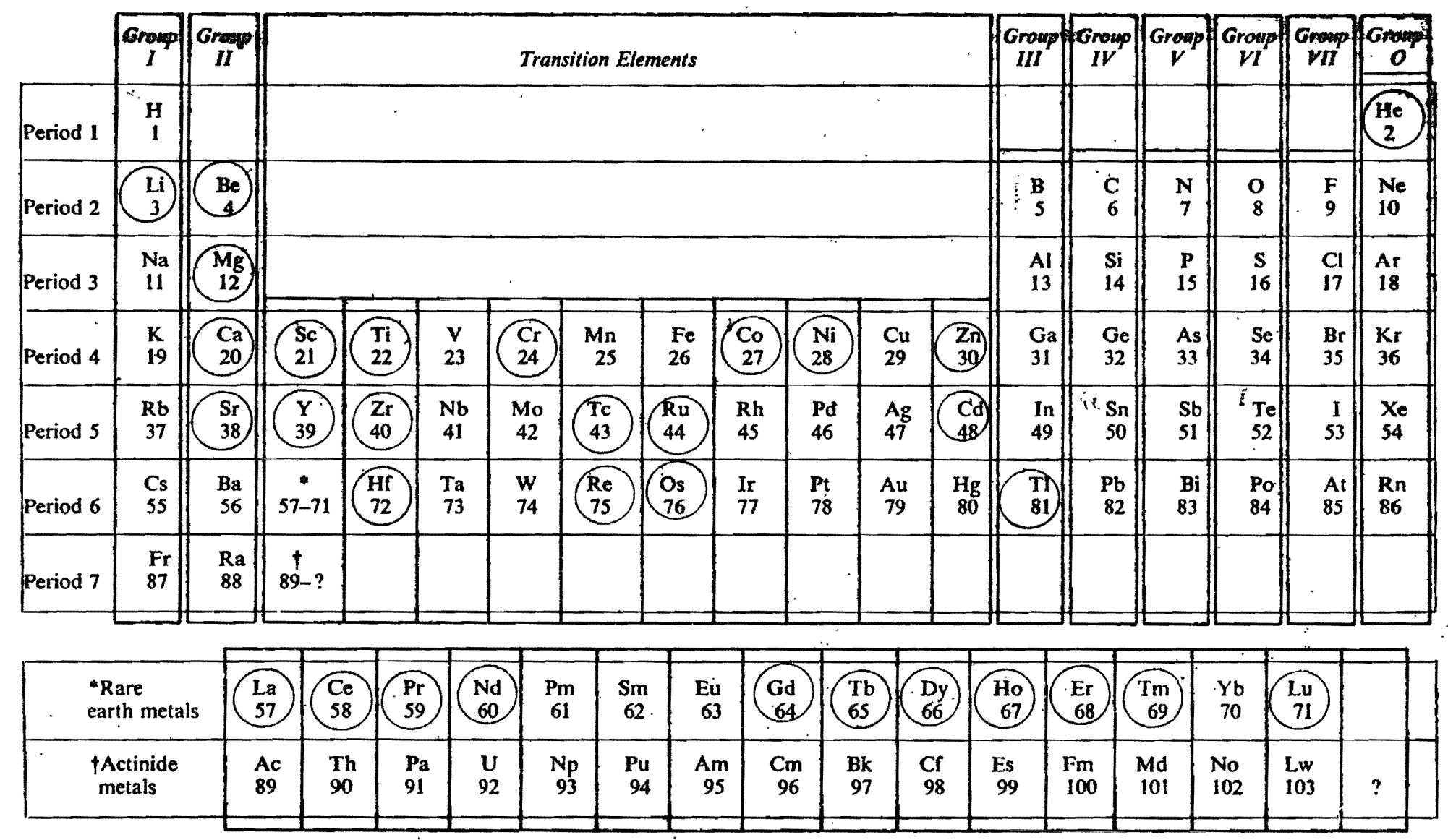

Figure 5. Positions of hcp structures in the periodic table of elements (indicated by circlès). 